\title{
Theory of Single-Molecule Spectroscopy: Beyond the Ensemble Average
}

Eli Barkai

Department of Chemistry and Biochemistry, Notre Dame University, Notre Dame, Indiana 46556; email: jbarkai@nd.edu

Younjoon Jung

Department of Chemistry, University of California, Berkeley, California 94720-1460;

email:younjoon@gold.cchem.berkeley.edu

\section{Robert Silbey}

Department of Chemistry, Massachusetts Institute of Technology, Cambridge, Massachusetts02139; email: silbey@mit.edu

Abstract Single-molecule spectroscopy (SMS) is a powerful experimental technique used to investigate a wide range of physical, chemical, and biophysical phenomena. The merit of SMS is that it does not require ensemble averaging, which is found in standard spectroscopic techniques. Thus SMS yields insight into complex fluctuation phenomena that cannot be observed using standard ensemble techniques. We investigate theoretical aspects of SMS, emphasizing (a) dynamical fluctuations (e.g., spectral diffusion, photon-counting statistics, antibunching, quantum jumps, triplet blinking, and nonergodic blinking) and (b) single-molecule fluctuations in disordered systems, specifically distribution of line shapes of single molecules in low-temperature glasses. Special emphasis is given to single-molecule systems that reveal surprising connections to Lévy statistics (i.e., blinking of quantum dots and single molecules in glasses). We compare theory with experiment and mention open problems. Our work demonstrates that the theory of SMS is a complementary field of research for describing optical spectroscopy in the condensed phase.

\section{INTRODUCTION}

Optical probing of single molecules (1-3), ions (4), nitrogen-vacancy centers (5), or quantum dots (QD) (6), in condensed phase or biological environments (7-9), is becoming routine practice in many laboratories. This new approach provides significant new insights into the interaction of light with matter, behavior of single particles, and their interaction with their nanoenvironments. For example, this method has shed light on mechanisms of single-protein folding (10-13).

Several excellent reviews have considered experimental aspects of SMS (3, 7-9, 14-16). In this review we discuss theoretical aspects of SMS. One of our 
aims is to emphasize the importance of fluctuations in SMS. Specifically, we analyze different physical and chemical mechanisms that are responsible for the fluctuation of the number of photon counts from single-molecule (SM) sources. We also recently surveyed the current status of the theory of SMS (17).

Why should we care about fluctuations in SMS? The merit of this new technique is that the problem of ensemble averaging, found in standard spectroscopic techniques, is totally removed. Hence, in many cases, SMS reveals fluctuation phenomena that are totally obscured by conventional measurement techniques (e.g., fluctuations in photon counts, photon antibunching, bunching, blinking, quantum jumps, spectral jumps, etc.). Analysis of these fluctuations reveals important information on dynamical processes occurring in the condensed phase as well as important information on the distribution of nanoenvironments. Many theoretical studies have yielded methods to analyze stochastic fluctuations of light intensity from single-molecule (SM) sources (e.g., see References 18-27). After an introduction to some of the concepts, methods, and mathematical tools used in the theory of SMS (see Sections 2 and 3), we review five types of fluctuation phenomena in SMS.

In Section 4, we briefly review the rate equation approach to SMS. This approach is important for the analysis of certain chemical behaviors of the molecules, for example, blinking behavior caused by the triplet state. This blinking exhibits the so-called quantum jump, where the molecule jumps into a nonemissive state (a dark state). Similar behavior occurs for single atomic systems (28). The mathematical theory of this behavior is simple to follow and is based on rate concepts.

In Section 5, we discuss a generalized Wiener-Khintchine approach $(29,30)$, which gives a mathematical framework for the calculation of single-molecule (SM) line-shape fluctuations. More precisely the method $(29,30)$ yields Mandel's $Q$ parameter describing the variance of number of emitted photons in terms of a three time dipole correlation function. The generalized Wiener-Khintchine approach $(29,30)$ is based on two main approximations: $(a)$ linear response theory, implying that external driving fields are weak and $(b)$ the semiclassical approach to photon-counting statistics. The calculation of the absorption line shape for a molecular electronic transition coupled to its environment is a classical problem in theoretical physical chemistry. When the excitation of the laser field is not strong, the calculation can be based on the Wiener-Khintchine theorem, i.e., the Fourier transform of the dipole relaxation function yields the line of the molecule. Although this fundamental approach is very useful in the context of SMS, it neglects the time-dependent fluctuations of the line (e.g., intensity fluctuations and fluctuations in a number of photon counts). These stochastic time dependencies are not found when the spectra of a large number of molecules are measured because they are averaged out in the ensemble measurement procedure. Thus, a fundamental problem in SMS is the analysis of time-dependent line-shape fluctuations. And the Generalized Wiener-Khintchine approach yields a method for the calculation of these fluctuations.

In Section 6, we briefly introduce the quantum-jump approach and emphasize the role of quantum mechanical fluctuations in SMS, namely sub-Poissonian 
photon statistics and antibunching. We briefly review the problem of single photons on demand using SM sources as an application of this approach. SM interacting with external fields are open dissipative quantum systems, which in many cases strongly interact with their nanoenvironments. A powerful theoretical approach called the quantum-jump approach was introduced in the quantum optics community to describe single experimental realizations of simple quantum systems (i.e., two-level atoms) $(28,33,34)$. An important ingredient of this theory is the concept of quantum jump. For our purposes, the quantum jump corresponds to the detection of a single photon emitted from an SM source. Such a jump corresponds to a sudden increase in the knowledge we have of the system, namely, the collapse of the wave function to the ground state following an emission of a single photon. The questions here are whether, when, and how to adopt the quantum-jump approach in the context of SMS. A step in this direction is the work of Makarov \& Metiu $(35,36)$ and the experiments and simulations of Orrit and co-workers (37).

Very recently, Zheng \& Brown $(31,32)$ developed a generating function framework to calculate photon statistics for an SM undergoing a spectral diffusion process. The approach in Reference 31, captures the quantum behavior of the interaction of light with matter within the framework of optical Bloch equations (e.g., antibunching) and is also valid for strong external fields. We briefly discuss this interesting work in Section 7.

In many cases, SMS reveals unexpected behavior that to date lacks theoretical explanations. One such example is the power-law statistical behavior of "dark" and "bright" times (i.e., blinking) of single quantum-dot systems (38-41). These systems are nonstationary and nonergodic, and they exhibit statistical aging (42, 43). These behaviors are related to the observation that the mean, dark, and bright periods diverge. As such, standard theories based on rate concepts, which work well for standard ensemble-averaged measurements, must be replaced when confronted with new fractal data obtained using SMS. In Section 8, we discuss a Lévy walk approach to the photon-counting statistics from such quantum dots. We showed that the Mandel's $Q$ parameter, describing fluctuation of photon counts, increases with measurement time even with long measurement times (42). This behavior is very different from that revealed by previous approaches, developed in the quantum optics community [e.g., $(28,87)$ and references therein], where Mandel's Q parameter approaches a constant in the limit of long measurement time.

In Section 9, we investigate SMS in low-temperature glasses as a prototype problem for SMS in a disordered medium. In a disordered condensed phase [e.g., low-temperature glasses, bacterial antena system LH2 $(45,46)$, and defected crystal], each SM interacts with a unique nanoenvironment. Hence, the spectra of an individual SM have unique features [see e.g., $(44,47,48)]$. Therefore, in a disordered host, one must consider many SM whose features are investigated individually. This leads to a distribution of parameters, for example, a distribution of lifetimes (49) or a distribution of moments or cumulants of the spectral lines $(50,51)$. These distribution functions contain more information than is 
obtained from the average alone, which is measured using ensemble techniques (e.g., averaged lifetime, averaged line shape, etc.). This leads to an important theoretical consequence: Predictions must be made on the level of distribution of observables, not just on the mean. A characteristic example is the Geva-Skinner model (44) for SM line shapes in low-temperature glasses. Both theory $(44,50)$ and experiment $(48,52)$ show that the line shape of an SM in a low-temperature glass typically has a complicated, multipeaked, random shape.

\section{FROM ENSEMBLE-AVERAGED LINE SHAPES TO SINGLE-MOLECULE LINES}

Here consider an SM immersed in a condensed-phase environment that we call the host (e.g., an amorphous glass, a molecular matrix, a cell, etc.). We introduce a simple picture for absorption spectroscopy in the condensed phase, which helps us to understand the new types of problems found in SMS. We emphasize some of the main differences between standard ensemble-averaged line-shape theories and SM behavior.

In SMS, one is interested in a particular electronic transition, whose states are denoted by $|g\rangle$ (ground state) and $|e\rangle$ (excited state). We neglect, for the time being, the triplet state, soon to be reintroduced. SM typically interacts strongly with their host. For simplicity, we assume that all molecular-host potentials $(a)$ are pair-wise additive and $(b)$ depend only on the distance between molecules. Although these assumptions are not always realistic, they are used here for the sake of clarity. The $\mathrm{SM} /$ host-molecule pair potential, when the SM is in the ground (excited) state is denoted by $v_{g}^{i}(r)\left[v_{e}^{i}(r)\right]$, where $i$ is an index for the $i$ th host molecule. The pair potential between $i$ th and $j$ th host molecules is $v_{h}^{i j}\left(r_{i j}\right)$. The Hamiltonian for the system with the molecule in the ground state is

$$
H_{g}=\frac{P^{2}}{2 M}+\sum_{i} \frac{p_{i}^{2}}{2 m_{i}}+\sum_{i} v_{g}^{i}\left(r_{i}\right)+\sum_{i<j} v_{h}^{i j}\left(r_{i j}\right) .
$$

The Hamiltonian when the SM is in the electronically excited state is

$$
H_{e}=H_{g}+\hbar \omega[\mathbf{R}(t)],
$$

where

$$
\hbar \omega[\mathbf{R}(t)] \equiv \hbar \omega_{0}+\sum_{i}\left[v_{e}^{i}\left(r_{i}\right)-v_{g}^{i}\left(r_{i}\right)\right] .
$$

$[\mathbf{R}(t)]$ denotes the collection of all time-dependent SM/host-molecule distances, and $\omega_{0}$ is the bare gas-phase transition frequency.

For a disordered system, the Hamiltonian Equation 1 is a random function. For example, consider a crystal host with quenched static defects that are randomly distributed in the sample with a low density. Each SM (which may also be considered a defect) will interact with a random set of host defects. For an ordered 
single-component host system, the Hamiltonian Equation 1 is simplified. Then $v_{g}^{i}(r)=v_{g}^{k}(r)$ for all $k \neq i$.

Consider an SM in the ground state. The absorption frequency of the molecule (i.e., $\left.\omega_{0}+\omega[\mathbf{R}(t)]\right)$ evolves in time according to the ground-state Hamiltonian. Such an evolution typically yields a complicated trajectory of the absorption frequency, called spectral diffusion.

In most cases, the dynamics of the underlying Hamiltonian is too complicated to track (or the Hamiltonian is not known). Then a useful approach is based on the Kubo-Anderson (53-55) stochastic theory, which replaces the absorption frequency $\omega[\mathbf{R}(t)]$ with a time-dependent stochastic process $\omega(t)$.

Consider now the classical absorption line-shape problem for such a molecule in a continuous wave (cw) weak-laser field. The line is given by the Fourier transform of the dipole correlation function, namely the Wiener-Khintchine formula:

$$
\left\langle I\left(\omega_{L}\right)\right\rangle \propto \int_{-\infty}^{\infty} \mathrm{d} t \exp \left[-i\left(\omega_{L}-\omega_{0}\right) t-\Gamma t / 2\right]\left\langle\exp \left\{i \int_{0}^{t} \omega\left[\mathbf{R}\left(t^{\prime}\right)\right] \mathrm{d} t^{\prime}\right\}\right\rangle,
$$

where $\langle\cdots\rangle$ indicates $(a)$ an equilibrium phase-space average over initial conditions weighted by the Boltzmann factor for the ground-state Hamiltonian or $(b)$ an average over the stochastic processes $\omega(t)$. $\Gamma$ is the inverse lifetime of the electronic transition.

In a disordered condensed phase, one has to add the averages over the random Hamiltonian to the average over the trajectories in Equation 4. Such randomness leads to the problem of inhomogeneous line broadening as reviewed by Stoneham (56). In effect, each individual chromophore has a random Hamiltonian caused by random defects in the sample. Hence, the absorption line shape observed in ensemble measurements is formally

$$
\text { line }=\overline{\left\langle I\left(\omega_{L}\right)\right\rangle},
$$

where $\cdots$ is an average over the random Hamiltonian.

The merit of SMS is that it gets rid of these two averages. Under certain conditions, discussed in Sections 3.1 and 5, SMS detects stochastic realizations of the spectral trajectories $\omega[\mathbf{R}(t)]$. The problem of inhomogeneous line broadening is removed. Hence, new problems are encountered: (a) What are the time-dependent fluctuations of the line of an SM? (b) In a disordered medium, what is the distribution of the line shape? The former is considered in Sections 3 through 8, and the latter is discussed in Section 9.

\section{METHODS AND CONCEPTS}

In Figure 1 we show a schematic representation of an electronic energy-level structure of an isolated SM. In a typical experiment, a cw laser (frequency $\omega_{L}$ ) excites the molecule; then, after the absorption of an exciting photon, the excited 


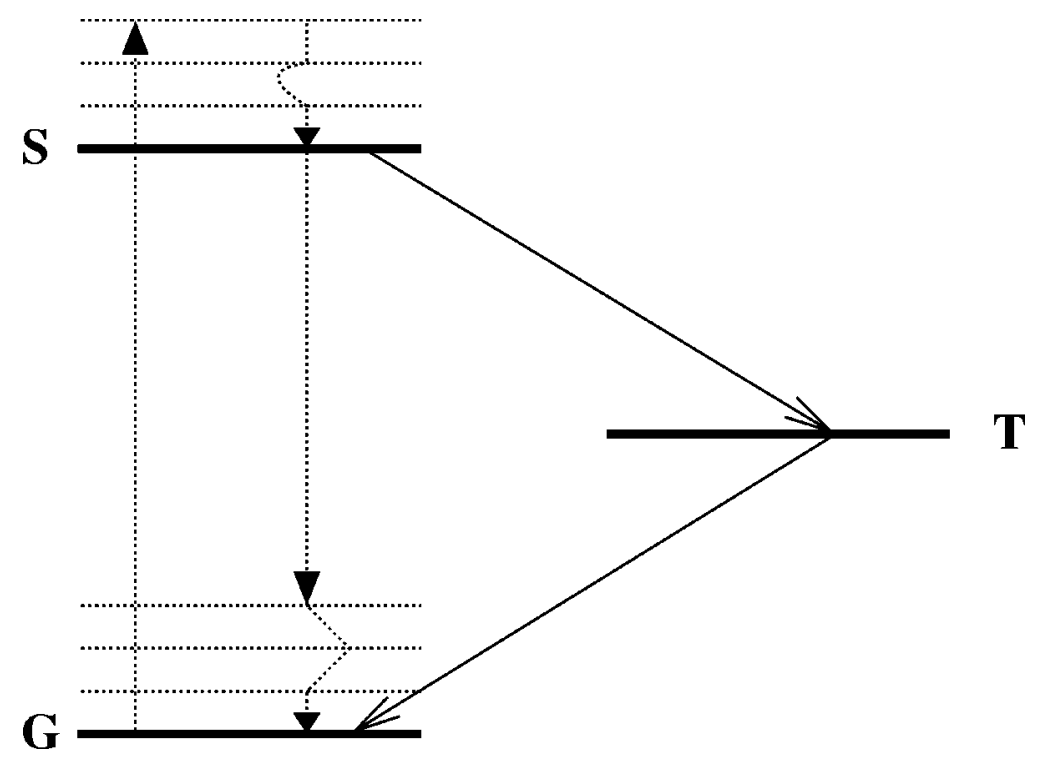

Figure 1 The three-state model of a static SM. The molecule in its ground state is excited to a vibrational level of the excited singlet state, and after a very fast vibrational relaxation, the molecule is found in the ground vibrational of the first electronic-excited state (S). Then, either the molecule emits a photon (dotted line pointing downward) or the system relaxes to the (typically) long-lived triplet state $(\mathrm{T})$. The fluorescent photons are Stokes-shifted with respect to the laser photons, owing to the vibrational levels. Hence, in experiment, one may distinguish between incoming laser photons and fluorescent photons.

molecule quickly decays (generating phonons) to the first electronic-excited state, from which a fluorescence photon is emitted. This cycle restarts once the system relaxes to the electronic ground state (see further discussion in Reference 3). In most (but not all) experiments involving SM, the fluorescence is simply collected and little concern is given to the finer details of the fluorescence frequency and spatial distributions.

SM spectra are extremely sensitive to the local nanoenvironment in which the SM is situated so that the spectra of the SM will fluctuate in time owing to changes in local environments. SMS takes advantage of this sensitivity by using $\mathrm{SM}$ as local probes. When the experiments are interpreted with proper theoretical modeling, they yield insight into the dynamics of the environment and the SM-bath interaction.

Mechanisms responsible for time-dependent fluctuations of SMS include tripletstate dynamics (57-59), blinking due to charging effects $(38,39)$, rotational dynamics $(60,61)$, and conformational changes in macromolecules $(8,20$, 
62-65). In some cases, relaxation rates describing SM dynamics, fluctuate in time. For example, the radiative lifetimes of quantum dots (66), or the triplet lifetime of SM in a polymer host (59), are time-dependent. Brown (67) considered a general approach to the theoretical study of SM kinetics with time-dependent rate constants. Here, we illustrate some characteristic time-dependent fluctuations of SMS, using spectral diffusion as an example.

\subsection{Spectral Diffusion and Spectral Trails}

We consider an SM undergoing a spectral diffusion process. Spectral diffusion is found in many SM systems $(7,9,52,62,63,68-74)$ and has been the subject of considerable theoretical work $(21,29,30,50,77-83)$. Perturbers such as two-level systems (TLS) or excitations in the environment lead to time-dependent random changes in the absorption frequency of the SM so that the SM is coming in and out of resonance with the fixed exciting laser field.

Although spectral diffusion processes were investigated in the context of ensemble-averaged linear and nonlinear spectroscopy $(55,75,76)$, the first observation of a stochastic realization of spectral diffusion was made by Moerner and coworkers $(68,69)$ using SMS of pentacene in p-terphenyl at $1.5 \mathrm{~K}$ (see Reference 84 for molecular modeling of this system). Reilly \& Skinner $(77,78)$ suggested a stochastic model on the basis of defect dynamics in the crystal that explains the microscopic origin of the spectral diffusion in this system.

In some cases, spectral diffusion is photoinduced (e.g., Reference 85). Recently, Bordat \& Brown (86) performed an empirical calculation of p-terphenyl crystal doped with terrylene to gain insight into the photoinduced frequency jumps of single terrylene molecules.

Using a generic stochastic approach, Kubo \& Anderson (53-55) investigated spectral diffusion processes occurring in condensed-phase environments. This approach is a useful tool for describing different types of line-shape phenomena. We used such an approach to describe an SM undergoing a spectral diffusion process. Following previous results (30), we assume that the absorption frequency of the molecule is $\omega=\omega_{0}+v h(t)$ and $h(t)$ describes a two-state dichotomic process [i.e., $h(t)=+1$ or $h(t)=-1$ ]. We assume the transition from state +1 to -1 and vice versa is described by a simple Poisson process defined by the rate $R$. In Sections 5 and 7 we use this simple two-state process to demonstrate characteristic behaviors of SM line-shape fluctuations. The physical meaning of rate $R$ and the frequency shift $v$ are discussed in Section 9 in the context of SMS in low-temperature glasses.

We first consider a slow-modulation case $R \ll \Gamma \ll v$, where $\Gamma$ is the inverse radiative lifetime of the molecule. Such a case corresponds to a large frequency shift and is sometimes called the inhomogeneous limit (although in the SM context, this terminology may be slightly misleading). Using the semiclassical theory of photon-counting statistics, we use the simple two-state process to generate spectral 
trails (for further details see Reference 30). A spectral trail is a stochastic twoor three-dimensional plot of the spectra of an SM $(9,52)$. In Figure 2 we show an example of such a trail, which is generated by dividing the time axis into bins of length $T$. During each bin interval one must determine the number of photon counts [which in our model (30) depends on the Rabi frequency $\Omega$; the stochastic realization of $h(t), \Gamma$, and the laser detuning $\left.\omega_{L}-\omega_{0}\right]$.

The spectral trail in Figure 2 illustrates the huge potential of SMS. Clearly, analysis of such a trail yields information on spectral-jump magnitudes, and statistics of spectral times. Such direct information cannot be obtained using ensembleaveraged techniques. We used several important conditions to obtain the spectral trail in Figure 2. First $R \ll v$; otherwise, we reach the limit of fast modulation exhibiting motional narrowing behavior (Figure 3). Second, $\Gamma \ll v$; otherwise the width of the spectral lines in Figure 2 will be larger than the spectral shift. Finally, $1 / R>T$; if not, then we do not sample the fluctuation of the line. $T$ must also be large enough so one may detect a sufficient number of photons in each bin. These restrictions typically limit the analysis of line-shape fluctuations, using spectral trails, to slow processes where average time between spectral jumps is on the order of milliseconds or seconds.

In Figure 3, we show the spectral trail of an SM undergoing a fast spectral diffusion process $v \ll R$. Unlike the slow modulation case, the spectral trail does not follow the absorption frequency of the molecule. An interesting question is whether careful analysis of photon statistics, emitted from such an SM source, can be used to teach us something about the underlying spectral diffusion process. The relation between photon-counting statistics and spectral diffusion is discussed in Section 5.

The spectral diffusion process considered here is an example of a time-dependent fluctuation in SMS. An important theoretical goal in this emerging field is understanding how to relate time-dependent fluctuations, such as intensity fluctuations, or fluctuations in the number of photon counts to the underlying processes occurring in the SM condensed-phase system. We emphasize the fluctuations because they constitute the basic new ingredient of SMS (i.e., in ensemble techniques these fluctuations are not observed because they are averaged out). Physically, the time-dependent fluctuations are controlled by three sources: the environment (e.g., spectral diffusion), intrinsic dynamical processes occurring in the molecule (e.g., triplet-state blinking), and quantum fluctuation of the SM laser-field system (e.g., antibunching, etc.). The following sections investigate time-dependent fluctuations in SMS.

\subsection{Mathematical Tools and Definitions}

The fluorescent photons emitted by a driven SM source are characterized by a mathematical formalism developed in the quantum optics community (87). Because this formalism has not been widely used by chemists, we take some time to introduce basic definitions. In what follows we assume a perfect detecting 
device; thus, we do not distinguish between photoemission events and photoelectron counts. Finite detection efficiency corrections to our results can be found in more specialized literature $[(30,87-89)$ and references therein]. These corrections are of practical importance; however, for the sake of clarity and space, we do not consider them here.

Photon emission is characterized in terms of a second-order correlation function: $g^{(2)}(t, t+\tau)$. This is the joint probability $P_{2}(t, t+\tau)$ for photon counts in the interval $[(t, t+\mathrm{d} t)$ and $(t+\tau, t+\tau+\mathrm{d} t)]$, normalized by the probability for two independent counts:

$$
g^{(2)}(t, t+\tau)=\frac{P_{2}(t, t+\tau)}{P_{1}(t) P_{1}(t+\tau)},
$$

where $P_{1}(t) \mathrm{d} t$ and $P_{1}(t+\tau) \mathrm{d} t$ are the probabilities for single-photon counts in the interval $(t, t+\mathrm{d} t)$ and $(t+\tau, t+\tau+\mathrm{d} t)$, respectively. Stationary emission processes are usually considered so that

$$
g^{(2)}(t, t+\tau)=g^{(2)}(\tau),
$$

which is $t$ independent.

The fluorescent photons emitted by a coherently driven SM exhibit the nonclassical effect of antibunching, namely

$$
\lim _{\tau \rightarrow 0} g^{(2)}(\tau)=0 .
$$

Physically, the emission of one photon from a single emitter makes the detection of a second photon, after a short delay, improbable (90). In the long time limit, one expects that

$$
\lim _{\tau \rightarrow \infty} g^{(2)}(\tau)=1
$$

because for long delay times the emission of two photons (not necessarily consecutive photons) is uncorrelated. Surprisingly, single quantum-dot spectroscopy has revealed a new type of strongly nonstationary behavior where Equation 9 does not hold. This is the topic of Section 8.

For an ensemble of SM one usually encounters a different behavior for $g^{(2)}(\tau)$. In many cases $g^{(2)}(\tau) \geq 1$. In this case we say that the light source is bunched. When statistics of emitted photons is Poissonian, $g^{(2)}(\tau)=1$.

In some experiments fluorescent photons are collected in time bins. A timedependent intensity $I(t)$ (i.e., photon counts per bin time) is obtained when the bin timescale is shorter than the timescale of the underlying physical/chemical processes responsible for the intensity fluctuations. In this case an intensity-intensity correlation function

$$
g_{I}^{(2)}(\tau)=\frac{\langle I(t+\tau) I(t)\rangle}{\langle I\rangle^{2}}
$$


is used to analyze dynamical processes in SMS. For example, Orrit and coworkers $(70,71)$ used this method to detect the dynamical process of an SM in a low-temperature glass; the timescale for the dynamical process was measured in microseconds.

Another useful quantity to characterize fluctuations is Mandel's $Q$ parameter (87):

$$
Q=\frac{\left\langle n^{2}\right\rangle-\langle n\rangle^{2}}{\langle n\rangle}-1,
$$

where $n$ is the random number of photon counts. When the statistics of photon counts is Poissonian, $Q=0$. The case $Q<0$ corresponds to sub-Poissonian statistics, whereas $Q>0$ corresponds to super-Poissonian statistics. For stationary processes there is a simple relation between $g^{(2)}(\tau)$ and Mandel's $Q$ parameter (e.g., Reference 28):

$$
Q(T)=\frac{2\langle I\rangle}{T} \int_{0}^{T} \mathrm{~d} t_{1} \int_{0}^{t_{1}} d t_{2} g^{(2)}\left(t_{2}\right)-\langle I\rangle T,
$$

where $T$ is the measurement time. Using $g^{(2)}(\tau)$ or $Q$ one may detect antibunching behavior $(5,91,92,94)$; the typical timescale is measured in nanoseconds. As far as we know, SMS has not been used to detect faster dynamics below the nanosecond limit.

\section{RATE-EQUATION APPROACH : TRIPLET BLINKING}

Fluorescence of molecules is often modeled using rate equations. This approach is the simplest and most widely used. Here we briefly discuss this approach in the context of SMS. Following Molski et al. (89), we consider a three-level molecule as a working example. A similar approach was used in References 92 and 93 in the analysis of the nonclassical photon statistics in SM fluorescence at room temperature (92). The rate-equation approach neglects spectral diffusion, coherence, and non-Markovian effects; these are discussed in the following sections.

Consider a three-level molecule, fixed in space, interacting with a cw laser field. We use a standard scheme and notation to describe the photon emission process of a chromophore, neglecting, for the time being, any explicit interaction with the environment. We label the states of the molecule with $i=G, T, S$ for the ground, triplet, and singlet states, respectively (95). The level diagram of the molecule is given in Figure 4. In this diagram, vibrational relaxation is neglected (see Figure 1). This is a reasonable assumption when the vibrational relaxation is extremely fast, compared with other timescales in the problem.

As investigated in detail by Barbara and co-workers (58), a three-level SM exhibits a blinking effect, sometimes called triplet blinking. Blinking is found in many SM systems. This means that long time intervals in which many photons are 


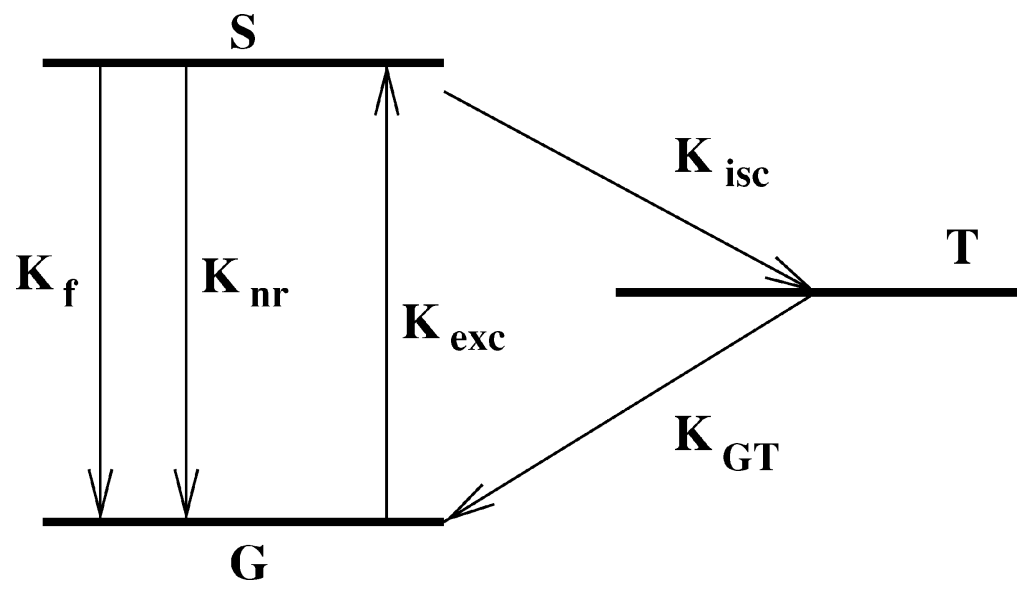

Figure 4 The three-state model for an SM. Five rate constants describe this problem, $K_{f}$, the fluorescence rate; $K_{n r}$, the nonradiative rate; $K_{\text {exc }}$, the excitation rate, $K_{i s c}$, the intersystem crossing; and $K_{G T}$, the decay rate from the triplet state.

emitted from the SM (bright state) are separated by long time intervals in which no photon is emitted (dark state). Because the molecule is not emitting photons when it is trapped in the triplet state, an observation of a single emitting molecule naturally leads to a blinking phenomenon. This behavior is easily detected when the timescale for the relaxation from triplet state is long (e.g., millisecond).

One goal of SM experiments is to extract information on the dynamical processes occurring on the level of an SM such as the rate constants appearing in Figure 4. Careful analysis of the photon statistics emitted from an SM should yield this type of information. In experiments the measurement time $T$ is bounded because SM photobleaches (96). In some cases this limits the collection of a sufficient number of counts to build up enough statistics (see, however, counter examples in References 97-99, where photostable SM were used).

In what follows we calculate some statistical properties of photons emitted from a single three-level system. We neglect the effect of photobleaching. We also assume an ideal detector and no background noise (see Reference 89 on these issues).

First, let us consider an ensemble of molecules. The populations $P_{i}(t)$ are described by the following rate equations (see Figure 4):

$$
\begin{aligned}
\frac{\mathrm{d}}{\mathrm{d} t} P_{G}(t) & =-K_{e x c} P_{G}(t)+\left(K_{f}+K_{n r}\right) P_{S}(t)+K_{G T} P_{T}(t), \\
\frac{d}{\mathrm{~d} t} P_{S}(t) & =K_{e x c} P_{G}(t)-\left(K_{f}+K_{n r}+K_{i s c}\right) P_{S}(t) \\
\frac{d}{\mathrm{~d} t} P_{T}(t) & =K_{i s c} P_{S}(t)-K_{G T} P_{T}(t)
\end{aligned}
$$


The solution of these equations using the Laplace transform technique is straightforward. The system of equations conserves normalization: $P_{G}(t)+P_{S}(t)+$ $P_{T}(t)=1$.

Whereas, for ensemble-averaged spectroscopy, the probabilities $P_{G}(t), P_{S}(t)$, and $P_{T}(t)$ are directly related to physical observables (i.e., the populations), new probability functions are needed to describe SM experiments. Let $f(\tau)$ be the probability-density function of times between consecutive emission events. CohenTannoudji \& Dalibard (100) call this function the delay function, but it is also called the waiting-time function. Because the molecule after an emission event is found in its ground state, $f(\tau)$ can be used to characterize the emission process. Once $f(\tau)$ is calculated, one may obtain other interesting characteristics of the emission process, for example, $g^{(2)}(\tau), Q$ (see further details in Section 6.2). Mathematically, the importance of $f(\tau)$ stems from the fact that the emission process is a renewal process $(101,102)$. Once a photon is emitted, the system returns to its ground state, and the process is renewed.

Molski et al. (91) showed how to calculate $f(\tau)$ for the three-level molecule under investigation. To calculate $f(\tau)$, a modified three-state model is introduced. An absorbing boundary condition, $A$, in Figure 5 is included in the modified scheme. This boundary "captures" the photon once it is emitted. Because there is no escape route from state $A$, the probability of a first-emission event is given by the occupation of this state. Hence, the following equations are used:

$$
\begin{aligned}
& \frac{d}{\mathrm{~d} t} \tilde{P}_{G}(t)=-K_{e x c} \tilde{P}_{G}(t)+K_{n r} \tilde{P}_{S}(t)+K_{G T} \tilde{P}_{T}(t), \\
& \frac{d}{\mathrm{~d} t} \tilde{P}_{S}(t)=K_{e x c} \tilde{P}_{G}(t)-\left(K_{f}+K_{n r}+K_{i s c}\right) \tilde{P}_{S}(t), \\
& \frac{d}{\mathrm{~d} t} \tilde{P}_{T}(t)=K_{i s c} \tilde{P}_{S}(t)-K_{G T} \tilde{P}_{T}(t) .
\end{aligned}
$$

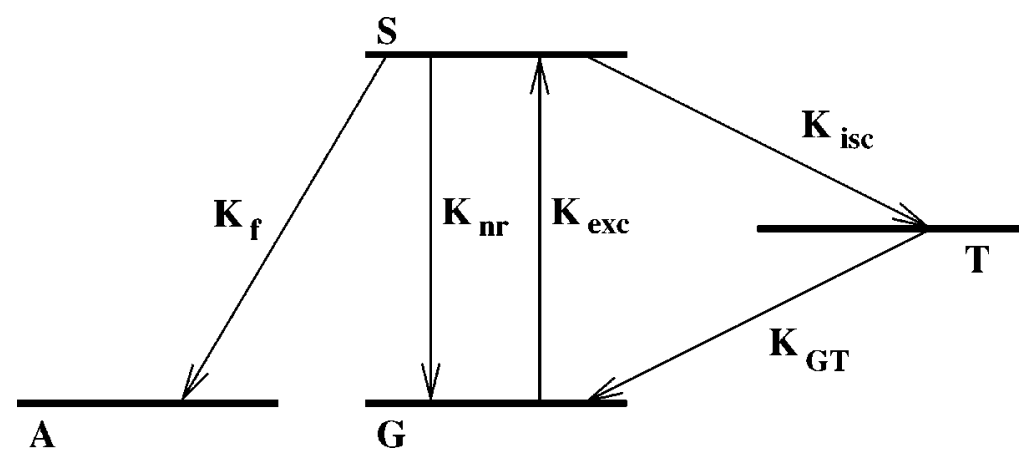

Figure 5 The three-state model for an SM with an absorbing boundary condition denoted with $A$. 
Note the difference between this set of equations and Equation 13. Owing to the absorbing boundary $\tilde{P}_{G}(t)+\tilde{P}_{S}(t)+\tilde{P}_{T}(t)<1$ for $t>0$, the waiting time function is $f(\tau)=K_{f} \tilde{P}_{S}(\tau)$ and $\tilde{P}_{S}(t)$ is determined by the set of Equation 14 with initial conditions $\tilde{P}_{G}(0)=1, \tilde{P}_{S}(0)=0$, and $\tilde{P}_{T}(0)=0$.

Using this scheme, it is easy to calculate the Laplace $(\tau \rightarrow u)$ transform of $f(\tau)$ :

$$
\hat{f}(u)=\frac{K_{e x c} K_{f}\left(K_{G T}+u\right)}{u^{3}+\Sigma u^{2}+\Pi u+K_{e x c} K_{f} K_{G T}},
$$

where $\Sigma=K_{n r}+K_{i s c}+K_{G T}+K_{f}+K_{e x c}$, and $\Pi=K_{e x c} K_{f}+K_{e x c} K_{G T}+$ $K_{f} K_{G T}+K_{e x c} K_{i s c}+K_{G T} K_{i s c}$. Finding the inverse Laplace transform of $\hat{f}(u)$ is straightforward. Here we illustrate a few points based on this equation.

Using the small $u$ expansion of Equation 15

$$
\hat{f}(u)=1-u\langle\tau\rangle+u^{2}\left\langle\tau^{2}\right\rangle / 2 \ldots,
$$

we obtain the first two moments of the waiting-time function. The averaged time between emission events

$$
\langle\tau\rangle=\int_{0}^{\infty} \tau f(\tau) \mathrm{d} \tau
$$

is

$$
\langle\tau\rangle=\frac{K_{e x c}\left(K_{G T}+K_{i s c}\right)+K_{G T}\left(K_{f}+K_{i s c}+K_{n r}\right)}{K_{e x c} K_{f} K_{G T}} .
$$

Using Equation 54, derived later, we find that Mandel's $Q$ parameter is in the long measurement time limit

$$
Q=\frac{2 K_{e x c} K_{f}\left(-K_{G T}^{2}+K_{e x c} K_{i s c}\right)}{\left[K_{e x c}\left(K_{G T}+K_{i s c}\right)+K_{G T}\left(K_{f}+K_{i s c}+K_{n r}\right)\right]^{2}} .
$$

Depending on parameters, $Q$ exhibits both sub- and super-Poissonian behaviors; however, we believe that in usual circumstances $K_{e x c}>K_{G T}^{2} / K_{i s c}$. As a result a super-Poissonian behavior $Q>0$ is encountered.

The simplest case to analyze is the two-level molecule, presented when $K_{i s c} \rightarrow 0$ assuming $K_{n r}=0$. For this case

$$
Q=-2 K_{e x c} K_{f} /\left(K_{e x c}+K_{f}\right)^{2}<0
$$

and

$$
f(\tau)=\frac{K_{e x c} K_{f}}{K_{f}-K_{e x c}}\left(e^{-K_{f} \tau}-e^{-K_{e x c} \tau}\right) .
$$

Hence, for short times

$$
f(\tau) \simeq K_{e x c} K_{f} \tau .
$$




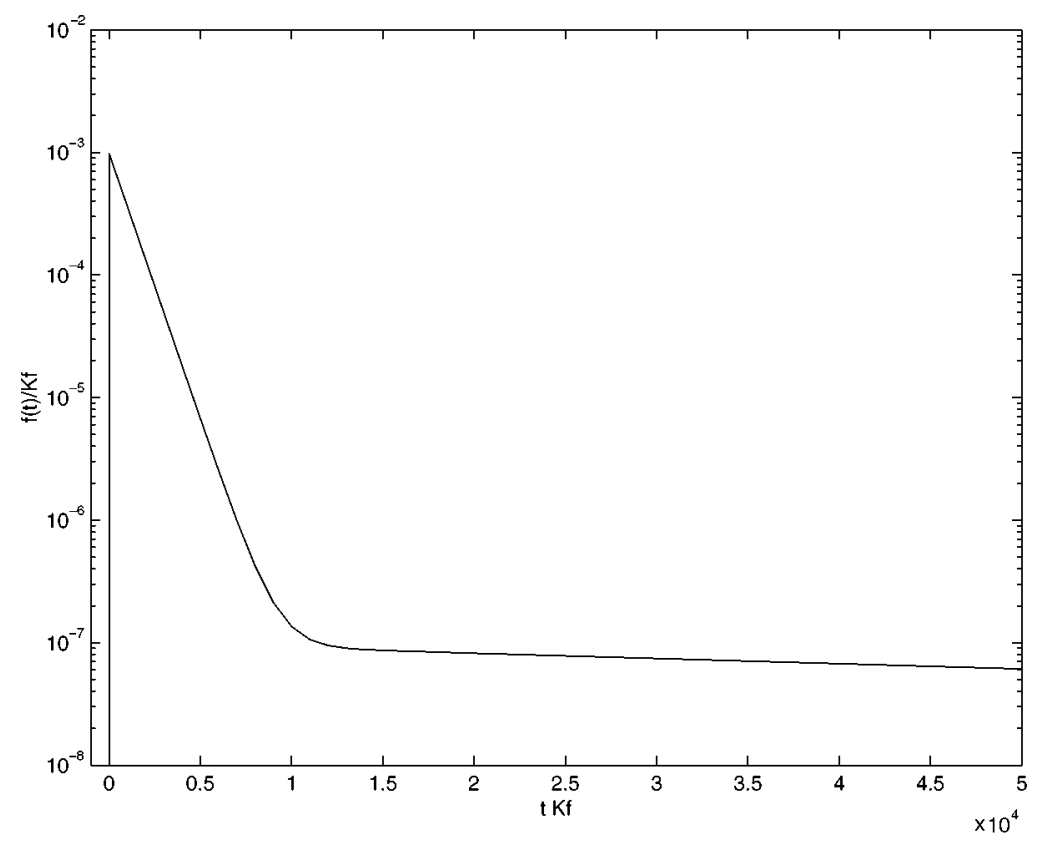

Figure 6 The semilog plot of the waiting-time probability-density function for a three-level SM. For very short times, $f(\tau)$ exhibits the effect of antibunching: $\left.f(\tau)\right|_{\tau=0}=0$. For longer times, $f(\tau)$ exhibits a biexponential behavior. The final slow decay of $f(\tau)$ is characteristic for systems exhibiting triplet blinking. $K_{f}=10^{8}, K_{i s c}=10^{6}, K_{e x c}=10^{5}, K_{G T}=10^{3}$, and $K_{n r}=0 \mathrm{~s}^{-1}$.

This is the mathematical manifestation of antibunching. We may say that a photon is delayed owing to the finite time it takes to excite the molecule; hence, $\left.f(\tau)\right|_{\tau=0}=0$.

In Figure 6 we show the waiting-time probability-density function $f(\tau)$ in a scaling form for a set of parameters satisfying $K_{f}>K_{i s c}>K_{\text {exc }}>K_{G T}>K_{n r}$. Three time-regimes are found. For very short times $K_{f} \tau \ll 1, f(\tau)$ exhibits antibunching. For longer times we observe a bi-exponential behavior. For $K_{f} \tau<$ $1\left(K_{f} \tau>1\right)$ we see a rapid (slow) decay of $f(\tau)$. The long-time behavior is caused by the long-lived triplet state of the molecule. While the molecule is in the triplet state, no photons are emitted (dark state). Hence, a typical sequence emitted from the molecule exhibits a triplet blinking effect. For the parameters under consideration, this case corresponds to a two-state molecule turning from bright to dark and vice versa.

The knowledge of the waiting-time function, $f(\tau)$, makes possible the calculation of the statistical properties of photons emitted from the SM (for further details see Section 6.2). The situation becomes more complicated when the molecule interacts with a time-dependent environment because then the renewal concept of the photon-emission process breaks down. In the next section we consider 
such a case: We investigate the effects of spectral diffusion on photon-counting statistics.

\section{THE GENERALIZED WIENER-KHINTCHINE THEOREM}

In many SM experiments one observes large fluctuations in the number of photon counts due to spectral diffusion. As mentioned in Section 3 perturbers in the vicinity of the molecule cause random spectral shifts in such a way that the absorption frequency of the molecule randomly fluctuates in time, leading to time-dependent spectra (as we demonstrated in the spectral trail in Figure 2). Here we investigate $\langle n\rangle$ and $Q$ for a model molecule undergoing a spectral diffusion process. We follow our work presented in References 29 and 30, where this problem was considered in detail.

We consider the semiclassical theory of photon-counting statistics. In this limit one of the most widely used approaches to line-shape theory is based on the Wiener-Khintchine theorem. This well-known theorem gives the line shape

$$
\left\langle I\left(\omega_{L}\right)\right\rangle=\lim _{T \rightarrow \infty} \frac{\langle n\rangle}{T}
$$

in terms of the complex Laplace transform of the molecule's dipole correlation function (see Equation 31 below). This fundamental theorem was used in a large number of theoretical investigations of line-shape phenomena $[(55,75,76)$ and references therein].

The merit of SMS is that it yields direct access to the measurement of the fluctuations of the line, e.g., $Q$. In what follows we generalize the Wiener-Khintchine theorem and show that, within linear response theory, $Q$ can be calculated on the basis of a three-time dipole-correlation function. This fundamental correlation function was obtained first by Plakhotnik (103) in the context of his intensityfrequency time-correlation function technique for SMS (104). A detailed analysis of the properties of the three-time correlation function and its relation to $Q$ was given in References 29 and 30. We will show how fluctuation in SMS, characterized by $Q$, can be used to obtain information on the underlying spectral diffusion process not contained in the line $\left\langle I\left(\omega_{L}\right)\right\rangle$.

In our investigation we assume fluctuations are caused by random modulations of the absorption frequency (i.e., spectral diffusion) that are treated stochastically, in a way similar to the Kubo-Anderson approach to line-shape theory. We neglect other physical mechanisms responsible for the line-shape fluctuation such as triplet-state dynamics, rotational dynamics, etc. We also assume semiclassical treatment that neglects the time ordering of the intensity operator (compare Equations 26 and 39). In Section 7 we treat the problem quantum mechanically, and then sub-Poissonian behavior $Q<0$ is found in certain limits. Further discussion of the physical and mathematical assumptions are given in References 29-32, and 124. 
The molecule in such cases is described by a $2 \times 2$ density matrix, $\rho$, whose elements are $\rho_{\mathrm{ee}}, \rho_{\mathrm{gg}}, \rho_{\mathrm{eg}}, \rho_{\mathrm{ge}}$. Thus

$$
\begin{aligned}
& u=\left(\rho_{\mathrm{ge}} e^{-i \omega_{\mathrm{L}} t}+\rho_{\mathrm{eg}} e^{i \omega_{\mathrm{L}} t}\right) / 2, \\
& v=-i\left(\rho_{\mathrm{ge}} e^{-i \omega_{\mathrm{L}} t}-\rho_{\mathrm{eg}} e^{i \omega_{\mathrm{L}} t}\right) / 2
\end{aligned}
$$

and

$$
w=\left(\rho_{\mathrm{ee}}-\rho_{g g}\right) / 2 .
$$

The stochastic Bloch equations, in the rotating wave approximation, are

$$
\begin{aligned}
\dot{u} & =\delta_{\mathrm{L}}(t) v-\Gamma u / 2, \\
\dot{v} & =-\delta_{\mathrm{L}}(t) u-\Omega w-\Gamma v / 2, \text { and } \\
\dot{w} & =\Omega v-\Gamma w-\Gamma / 2 .
\end{aligned}
$$

As previously mentioned, $\Gamma$ is the inverse lifetime of the molecule, and $\Omega$ is the Rabi frequency. The detuning frequency

$$
\delta_{\mathrm{L}}(t)=\omega_{\mathrm{L}}-\omega_{0}-\omega(t)
$$

is stochastic and time dependent (i.e., the spectral diffusion). Without loss of generality, we set the bare absorption frequency of the molecule: $\omega_{0}=0$.

According to the semiclassical theory of photon-counting statistics (87), the probability of recording $n$ photons in a time interval $(0, T)$ is given by $(87)$

$$
p(n, T)=\left\langle\frac{W^{n}}{n !} \exp (-W)\right\rangle,
$$

where the dimensionless variable

$$
W \equiv \xi \Omega \int_{0}^{T} v(t) \mathrm{d} t
$$

is the detection efficiency $\xi$ times the work of the driving field per unit energy (defined by $\hbar \omega_{\mathrm{L}}$ ). Using Equation 26, the average number of photons counted in time interval $(0, T)$ is

$$
\langle n\rangle=\langle W\rangle
$$

where $\langle\cdots\rangle$ is an average over the spectral diffusion process. Mandel's $Q$ parameter (Equation 11), is used to characterize the fluctuations, and it is straightforward to show that (87)

$$
Q=\frac{\left\langle W^{2}\right\rangle-\langle W\rangle^{2}}{\langle W\rangle}
$$


Here $Q \geq 0$, indicating that photon statistics are super-Poissonian. Equation 29 shows that fluctuations in work done by the laser field on the molecule lead to fluctuations in the number of photon counts.

We now consider the important limit of the weak cw-laser intensity of Equation 25 . We use a perturbation expansion in the Rabi frequency to find within linear response theory $(29,30)$,

$$
v=\frac{\Omega}{2} \operatorname{Re}\left\{\int_{0}^{t} \mathrm{~d} t_{1} \exp \left[-i \int_{t_{1}}^{t} \mathrm{~d} t^{\prime} \delta_{\mathrm{L}}\left(t^{\prime}\right)-\Gamma \frac{\left(t-t_{1}\right)}{2}\right]\right\} .
$$

In standard line-shape theories Equation 30 is averaged. The well-known result gives the Wiener-Khintchine formula for the line shape, using Equation 27

$$
\left\langle I\left(\omega_{L}\right)\right\rangle=\lim _{T \rightarrow \infty} \frac{\langle W\rangle}{T}=\frac{\xi \Omega^{2}}{2} \operatorname{Re}\left[\int_{0}^{\infty} \mathrm{d} \tau e^{-i \omega_{L} \tau-\Gamma \tau / 2} C_{1}(\tau)\right],
$$

where

$$
\mathrm{C}_{1}(\tau) \equiv\left\langle e^{i \int_{0}^{\tau} \omega\left(t^{\prime}\right) \mathrm{d} t^{\prime}}\right\rangle
$$

is the one-time dipole-correlation function (i.e., a Kubo-Anderson expression for the dipole-correlation function).

We now consider the fluctuation using Equations 27 and 30

$$
\begin{aligned}
\left\langle W^{2}\right\rangle= & \frac{\xi^{2} \Omega^{4}}{16} \int_{0}^{T} \int_{0}^{T} \int_{0}^{T} \int_{0}^{T} \mathrm{~d} t_{1} \mathrm{~d} t_{2} \mathrm{~d} t_{3} \mathrm{~d} t_{4} \\
& \times e^{-i \omega_{\mathrm{L}}\left(t_{2}-t_{1}+t_{3}-t_{4}\right)-\Gamma\left(\left|t_{1}-t_{2}\right|+\left|t_{3}-t_{4}\right|\right) / 2} \mathrm{C}_{3}\left(t_{1}, t_{2}, t_{3}, t_{4}\right),
\end{aligned}
$$

where

$$
\mathrm{C}_{3}\left(t_{1}, t_{2}, t_{3}, t_{4}\right)=\left\langle\exp \left[i \int_{t_{1}}^{t_{2}} \omega\left(t^{\prime}\right) \mathrm{d} t^{\prime}-i \int_{t_{3}}^{t_{4}} \omega\left(t^{\prime}\right) \mathrm{d} t^{\prime}\right]\right\rangle .
$$

The correlation function in Equation 34 contains the information on the spectral diffusion process relevant for the calculation of $Q$ (i.e., using Equations 29 and $31)$.

The detailed analysis of SM line-shape fluctuations based on Equations 33 and 34 (References 29 and 30) leads to the following points:

1. The correlation function (Equation 34) is a four-time dipole-correlation function. However, if the underlying spectral diffusion process is stationary, it is given by a three-time correlation function. 
2. The correlation function has a structure similar to correlation functions found in the theory of nonlinear spectroscopy (75). Thus, fluctuations in the number of photon counts in linear SMS are mathematically related to nonlinear ensemble-averaged spectroscopy. Recently, Mukamel (105) extended our understanding of the relation between nonlinear spectroscopy and photoncounting statistics to the fully quantum domain.

3. There are 24 ways to time-order $t_{1}, t_{2}, t_{3}$, and $t_{4}$ in Equation 34; however, only three of these time-orderings are important.

4. $Q$ depends on the measurement time, $T$. However, unless specified otherwise, we consider only the steady-state limit $T \rightarrow \infty$ of $Q$. For time dependencies of $Q$, see References 29 and 30 .

\subsection{An Example}

To investigate the generic behavior of line-shape fluctuation, a simple two-state spectral-diffusion model was investigated in great detail $(29,30)$. The spectraldiffusion process considered was $h(t)=v h(t)$, where $h(t)$ is a telegraph process $h(t)=+1$ or -1 with the transition rate between these two states equal to $R$ as was used by Kubo in his classical investigation of line shape.

Using this model one may characterize SM fluctuations in six regimes. These depend on the relative magnitude of the parameters $v, R$, and $\Gamma$. In different regimes, $Q$ exhibits different physical behaviors.

5.1.1. SLOW MODULATION The most straightforward regime is the strong-coupling slow-modulation limit, $R \ll \Gamma \ll v$, where the spectral trail of the molecule follows the absorption frequency of the molecule (as in Figure 2). Using the generalized Wiener-Khintchine approach, we derived the following expression for the time-dependence of $Q$ :

$$
Q \simeq \frac{\xi}{2 R}\left(1+\frac{e^{-2 R T}-1}{2 R T}\right) \frac{\left(I_{+}-I_{-}\right)}{\left(I_{+}+I_{-}\right)},
$$

where

$$
I_{ \pm}=\frac{\Omega^{2} \Gamma\left|\Gamma / 2+i\left(\omega_{L} \mp \nu\right)\right|^{-2}}{4}
$$

are stationary solutions of the time-independent Bloch equation. In this limit the line is given by

$$
\left\langle I\left(\omega_{L}\right)\right\rangle \simeq \frac{I_{+}+I_{-}}{2} .
$$

Comparing the last two equations, we see that both $Q$ and $\left\langle I\left(\omega_{L}\right)\right\rangle$ exhibit splitting behavior, with two peaks at $\omega_{L}= \pm v$. However, unlike the line shape, $Q$ depends on time $T$ and the rate $R$. Thus, it is $Q$ and not $\left\langle I\left(\omega_{L}\right)\right\rangle$ that yields information on the dynamics. The behavior of both $\left\langle I\left(\omega_{L}\right)\right\rangle$ and $Q$ is shown in Figure 7. A generic 
feature is the decrease in $Q$ as the underlying spectral-diffusion process becomes faster.

The behavior found in the slow-modulation limit can be easily understood on the basis of a two-state random-walk model. The molecule can be found in either a positive $+v$ or negative $-v$ state. In the slow-modulation limit, the rate of photon emission is determined by the stationary solutions of the time-independent Bloch equation, namely $I_{ \pm}$, while transients may be neglected. Using this physical picture one can easily derive Equations 35 and 36.

5.1.2. FAST MODULATION We now consider the fast-modulation limit (a typical spectral trail is shown in Figure 3). In this limit the line is Lorentzian; its width is $\Gamma+\Gamma_{\text {eff }}$ with

$$
\Gamma_{\text {eff }}=\frac{v^{2}}{R} .
$$

As shown in Figure $8 a$ (see insert) the line exhibits the well-known behavior of motional narrowing, i.e., as $R$ is increased, the line width is decreased. When $\Gamma_{\text {eff }}=0$ (i.e., $R \rightarrow \infty$ ), the line width is determined by the lifetime of the excited level, namely $\Gamma$.

$Q$ exhibits either a Lorentzian or a splitting behavior depending on $\Gamma$ and $\Gamma_{\text {eff }}$ :

$$
Q \simeq\left\{\begin{array}{cl}
\frac{\xi \Omega^{2} \Gamma_{\text {eff }}}{2 \Gamma\left(\omega_{\mathrm{L}}^{2}+\Gamma_{\text {eff }}^{2} / 4\right)} & \Gamma \ll \Gamma_{\text {eff }} \\
\frac{\xi \Omega^{2} \Gamma_{\text {eff }} \Gamma \omega_{\mathrm{L}}^{2}}{\left(\omega_{\mathrm{L}}^{2}+\Gamma^{2} / 4\right)^{3}} & \Gamma_{\text {eff }} \ll \Gamma .
\end{array}\right.
$$

A detailed discussion of Equation 38 based on a cumulant and factorization approximations of the three-time dipole-correlation function is given in References 29 and 30. When $\Gamma_{\text {eff }}=0$ (i.e., $R \rightarrow \infty$ ), we have $Q=0$, i.e., Poisson statistics. The semiclassical behavior of $Q$ in other limits is summarized in Table 1 .

In the fast-modulation limit, and when $\Gamma_{\text {eff }}$ is finite, small deviations from Poisson statistics are found. For reasonable detection efficiency, a typical value of $Q$ is $10^{-4}$ (28), which is very difficult to detect in experiments [values of $|Q|$ as small as $10^{-4}$ have been detected in the context of antibunching (92)]. It is therefore important to consider the problem in the limit of strong external fields (124), for which linear response theory does not work (for details see Section 7).

The generalized Wiener-Khintchine approach yields the fluctuation of photon counts in the linear response regime. This approach is based on a semiclassical theory of photon-counting statistics (87). As such it neglects certain quantum aspects of interaction of light with matter $(29,30)$. For example, it is not suited for the description of antibunching and sub-Poissonian photon statistics. In the next section, we consider the quantum-jump approach, which yields the quantum evolution of single emitting objects in external fields. In Section 7 we consider the quantum mechanical treatment of an SM undergoing the spectral diffusion process, showing that for fast spectral-diffusion processes sub-Poissonian statistics describe the emission process. 
TABLE 1 Behavior of the line shape $\left\langle I\left(\omega_{L}\right)\right\rangle$ and $Q$ based on semiclassical generalized Wiener-Khintchine approach.

\begin{tabular}{|c|c|c|c|}
\hline & Slow modulation & Intermediate & Fast modulation \\
\hline Weak coupling & $R \ll v \ll \Gamma$ & $\begin{aligned} v \ll R & \ll \Gamma \\
\langle I\rangle & \sim \frac{\Omega^{2} \Gamma}{4\left[\omega_{L}^{2}+\Gamma^{2} / 4\right]} \\
Q & \sim \frac{\Gamma_{\mathrm{eff}}}{\langle I\rangle}\left(\frac{\mathrm{d}\langle I\rangle}{\mathrm{d} \omega_{L}}\right)^{2}\end{aligned}$ & $v \ll \Gamma \ll R$ \\
\hline Strong coupling & $\begin{array}{l}R \ll \Gamma \ll v: \text { case }(\mathrm{i}) \\
\langle I\rangle \sim \frac{1}{2}\left(I_{+}+I_{-}\right) \\
Q \sim \frac{\langle I\rangle}{R}\end{array}$ & $\begin{array}{l}\Gamma \ll R \ll v: \text { case }(\mathrm{v}) \\
\langle I\rangle \sim \frac{\Omega^{2} v^{2} R}{\left(\omega_{L}^{2}-v^{2}\right)^{2}+4 R^{2} \omega_{L}^{2}} \\
Q \sim \frac{2\langle I\rangle}{\Gamma}\end{array}$ & $\begin{array}{l}\Gamma \ll v \ll R: \text { case (iii) } \\
\langle I\rangle \sim \frac{\Omega^{2}\left(\Gamma+\Gamma_{\text {eff }}\right)}{4\left[\omega_{L}^{2}+\left(\Gamma+\Gamma_{\text {eff }}\right)^{2} / 4\right]} \\
Q \sim \begin{cases}\frac{2\langle I\rangle}{\Gamma} \quad \Gamma_{\text {eff }} \gg \Gamma \\
\frac{\Gamma_{\text {eff }}}{\langle I\rangle}\left(\frac{\mathrm{d}\langle I\rangle}{\mathrm{d} \omega_{L}}\right)^{2} \Gamma_{\text {eff }} \ll \Gamma\end{cases} \end{array}$ \\
\hline
\end{tabular}

*We set the detection efficiency $\xi=1$. The slow- and fast-modulation regimes are defined according to $R \ll v$ and $R \gg v$, respectively. The strong and weak coupling regimes are defined according to $v \gg \Gamma \Gamma \gg v$, respectively. Rich types of physical behaviors are shown. In the fast modulation limit a quantum mechanical treatment of the problem (124) yields $Q<0$, Equation 62, and then the semiclassical treatment of the problem breaks down.

\section{QUANTUM-JUMP APPROACH}

Usually a density matrix or rate equation is used to describe the time evolution of an ensemble of particles interacting with an external laser field. In recent years a powerful theoretical approach, based on non-Hermitian wave function evolution, was developed to describe single experimental realizations of single quantum systems. The approach, called the quantum-jump approach, was introduced to describe simple dissipative atomic systems interacting with a laser field (see References 28 and 107 for a review and historical account).

An important ingredient of this new approach is the concept of a quantum jump. For our purposes, the quantum jump corresponds to the detection of a photon emitted from an SM source. Such an event corresponds to a sudden change in the information we have on the system, namely to the collapse of the SM wave function to its ground state, following an emission of a photon. Thus, unlike the standard density matrix description of an open quantum system, the evolution of a single emitting object is not smooth.

An excellent review by Plenio \& Knight (28) is a good starting point for an introduction to the approach. In particular, Reference 28 gives valuable Monte Carlo methods which are used in simulation of the photon emission process from an SM source. Here we consider a simple problem that illustrates some of the basic ingredients of the new approach. Then we briefly discuss the applications of the quantum-jump approach in the context of SMS. 
Qualitatively speaking, the quantum-jump approach for SMS should be different from the quantum-jump approach employed by the quantum optics community for two simple reasons: First, molecules may exhibit a more complex behavior than simple two-level atoms. The work of Makarov \& Metiu $(35,106)$ examines this idea. Second, SM interacts with their condensed-matter environment, leading to stochastic time-dependent fluctuations not found for simple ideal isolated atomic systems. Much work in this direction is needed.

\subsection{Fluorescence Photon-Number Statistics}

We consider a two-level SM/atom interacting with a cw-laser field, within the rotating wave approximation, and neglect spectral diffusion and any other external noise source. A classical problem is the calculation of $p(n, T)$ : the probability that $n$ photons have been emitted by an SM in time interval $(0, T)$. This problem is usually solved using the counting formula $(88,108,109,113)$,

$$
p(n, T)=\left\langle\mathcal{T}:: \frac{1}{n !}\left[\int_{0}^{T} \mathrm{~d} t \hat{I}(t)\right]^{n} \exp \left[-\int_{0}^{T} \mathrm{~d} t \hat{I}(t)\right]\right\rangle_{Q M},
$$

where $\mathcal{T}$ is the time-ordering symbol, :: stands for normal ordering, \langle\rangle$_{Q M}$ represents the quantum mechanical expectation value for the total state of the TLS plus field, and $\hat{I}(t)$ is the operator for the total flux expressed in units of photons per unit time. Results for $p(n, T)(88,108,109,113)$, are re-derived here using the quantumjump approach. Below, we omit the subscript $\mathrm{QM}$ in \langle\rangle$_{Q M}$ (the reader should not confuse this average with the averaging over spectral diffusion discussed in the previous section).

The ground and excited state of the molecule are denoted by $|e\rangle$ and $|g\rangle$, respectively. The system is described by a non-Hermitian Hamiltonian

$$
H_{n H}=H_{M}-i \frac{\Gamma}{2}|e\rangle\langle e| .
$$

Here

$$
H_{M}=-\Delta|e\rangle\langle e|+\frac{\Omega}{2}(|g\rangle\langle e|+| e\rangle\langle g|),
$$

where $\Delta=\omega_{L}-\omega_{0}$ is the detuning and $\Gamma$ is the radiative decay rate of the molecule. The time evolution of the "wave function" is given by

$$
i|\dot{\Psi}\rangle=H_{n H}|\Psi\rangle .
$$

However, since $H_{n H}$ is non-Hermitian, $|\Psi\rangle$ is not normalized, $\langle\Psi \mid \Psi\rangle<1$ for $t>0$. The usual textbook interpretation of the wave function $|\Psi\rangle$ clearly does not hold. So, the fundamental question is, What is $|\Psi\rangle$ ?

According to the quantum-jump approach, $|\Psi\rangle$ yields the survival probability of the photon. $P_{0}(t)$ is the probability that a photon was not emitted in the time 
interval $(0, t)$. Then according to the quantum-jump approach,

$$
P_{0}(t)=\langle\Psi \mid \Psi\rangle .
$$

For the SM under consideration $|\Psi\rangle$ is given by

$$
|\Psi\rangle=\alpha(t)|g\rangle+\beta(t)|e\rangle,
$$

where initial conditions satisfy $|\alpha(0)|^{2}+|\beta(0)|^{2}=1$. The coefficients $\alpha(t)$ and $\beta(t)$ can be easily calculated using the non-Hermitian Hamiltonian Equation 40. Based on Equation 43

$$
\begin{gathered}
P_{0}(t)=|\alpha(t)|^{2}+|\beta(t)|^{2} \\
\frac{1}{\left|\Lambda_{+}-\Lambda_{-}\right|^{2}}\left[\left|\Lambda_{+} e^{\Lambda_{-} t}-\Lambda_{-} e^{\Lambda_{+} t}\right|^{2}+\Lambda_{+} \Lambda_{-}\left|e^{\Lambda_{+} t}-e^{\Lambda_{-} t}\right|^{2}\right]
\end{gathered}
$$

where

$$
\Lambda_{ \pm}=\frac{i \tilde{\Delta} \pm \sqrt{-\tilde{\Delta}^{2}-\Omega^{2}}}{2}
$$

and $\tilde{\Delta} \equiv \Delta+i \Gamma / 2$. Deriving Equation 45 we assumed that the system is initially in its ground state $|\Psi(t=0)\rangle=|g\rangle$.

Because a cw laser is acting on the SM, the molecule is excited, and it emits a photon at a random time. Then the processes are renewed. Quantum jumps occur at random times $\left\{t_{1}, t_{2}, \cdots t_{n} \cdots\right\} . \tau_{n}=t_{n+1}-t_{n}$ is the time interval between successive emission events. The random variables $\left\{\tau_{n}\right\}$ are mutually independent, identically distributed random variables. The waiting-time probability-density function describing these random variables is

$$
f(\tau)=-\frac{\mathrm{d} P_{0}(\tau)}{\mathrm{d} \tau}
$$

$\left(P_{0}(\tau)\right.$ is given in Equation 45). After each emission event the system is in the ground state, so the proper initial conditions are those used to derive Equation 45.

For zero detuning $(\Delta=0)$ :

$$
f(\tau)=\frac{4 \Gamma \Omega^{2}}{\left|\Gamma^{2}-4 \Omega^{2}\right|} e^{-\Gamma \tau} \sin h^{2}\left(\frac{\sqrt{\Gamma^{2}-4 \Omega^{2}}}{4} \tau\right),
$$

which is in agreement with Equation 30 (88). For weak laser fields $\Omega<\Gamma$, the system exhibits a damped behavior, whereas in the opposite limit $\Gamma<\Omega$, underdamped Rabi oscillations are found. As expected, antibunching yields $\left.f(\tau)\right|_{\tau=0}=$ 0 . Figures 9 and 10 show the behavior of the waiting-time probability-density function for weak and strong fields. At least in the strong-field limit, the behavior is different from the behavior observed in Figure 6 for a three-level molecule described by simple rate equations. The quantum mechanical calculation yields Rabi oscillations in the waiting-time distribution. Both Rabi oscillations and antibunching have been detected in SMS (91, 92, 94, 110-112). 


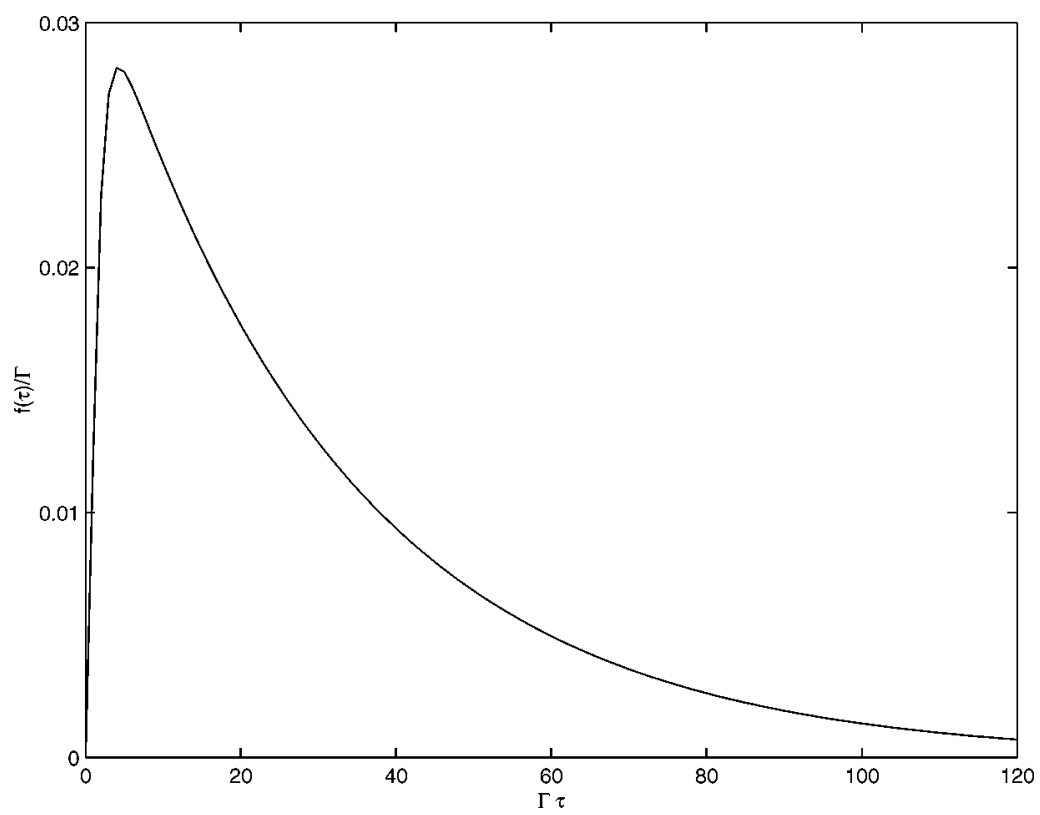

Figure 9 The waiting-time probability-density function for resonance fluorescence (zero detuning) in the overdamped limit $\Omega / \Gamma=0.25$. When $\tau \rightarrow 0, f(\tau)=0$, implying antibunching behavior. For longer times $f(\tau)$ decays exponentially.

\subsection{Renewal Theory Approach}

Once $f(\tau)$ is known, statistics of a number of quantum jumps/photon emissions can be calculated using renewal theory $(101,102)$.

$$
\hat{f}(u)=\int_{0}^{\infty} \exp (-u \tau) f(\tau) \mathrm{d} \tau
$$

denotes the Laplace transform of $f(\tau)$. The probability of $n$ emission events in a given time interval $(0, T)$ is easily calculated using the convolution theorem of the Laplace transform

$$
P_{n}(T)=\mathcal{L}^{-1}\left\{\frac{1-\hat{f}(u)}{u} \hat{f}^{n}(u)\right\}
$$

where $\mathcal{L}^{-1}$ denotes the inverse Laplace transform. Here the system is in a ground state at the start of the observation. Hence, statistics of waiting time for the first photon are given by $f(\tau)$. 


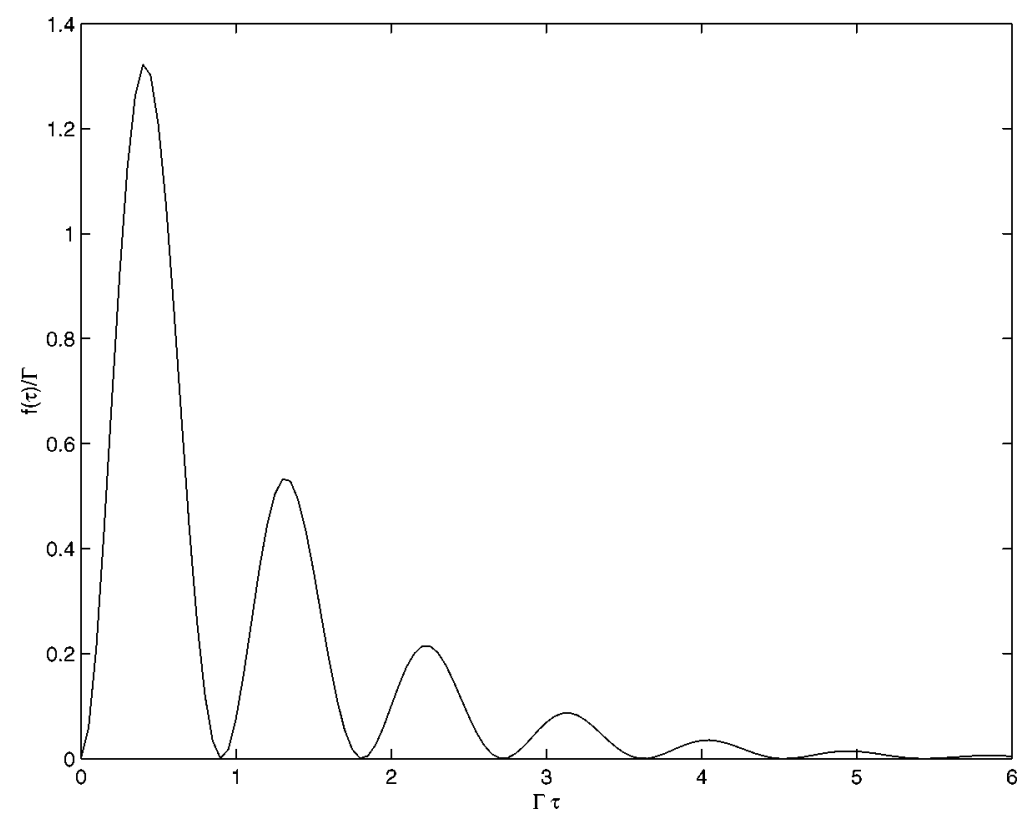

Figure 10 The waiting-time probability-density function for resonance fluorescence in the underdamped limit $\Omega / \Gamma=7$ with zero detuning. Rabi oscillations are observed, and $f(\tau)$ exhibits a nonexponential behavior.

Using Equation 50 the mean may be calculated as

$$
\langle\hat{n}(u)\rangle=\sum_{n=0}^{\infty} n \hat{P}_{n}(u)=\frac{\hat{f}(u)}{u[1-\hat{f}(u)]},
$$

and the fluctuation of photon counts is described using

$$
\left\langle\hat{n}^{2}(u)\right\rangle-\langle\hat{n}(u)\rangle=\frac{2 \hat{f}^{2}(u)}{u[1-\hat{f}(u)]^{2}} .
$$

Using the small $u$ expansion of these expressions, we obtain the large $t$ behavior of the mean

$$
\langle n\rangle \sim \frac{t}{\langle\tau\rangle}
$$

and of $Q$

$$
Q=\frac{\left\langle n^{2}\right\rangle-\langle n\rangle^{2}}{\langle n\rangle}-1 \sim \frac{\left\langle\tau^{2}\right\rangle-2\langle\tau\rangle^{2}}{\langle\tau\rangle^{2}} .
$$

Here 


$$
\langle\tau\rangle=\int_{0}^{\infty} \tau f(\tau) \mathrm{d} \tau
$$

and

$$
\left\langle\tau^{2}\right\rangle=\int_{0}^{\infty} \tau^{2} f(\tau) \mathrm{d} \tau
$$

are the first two moments of the probability-density function $f(\tau)$. From Equation $54,-1<Q$.

Cox (101) showed that for renewal processes, in the long time limit, $n$ is normally distributed with a mean $t /\langle\tau\rangle$ and variance $\left(\left\langle\tau^{2}\right\rangle-\langle\tau\rangle^{2}\right) t /\langle\tau\rangle^{3}$. This is a consequence of the Gaussian central-limit theorem. It shows that for long measurement times statistics of photons do not depend on the specific shape of the waiting-time distribution, only on the first two moments (or on the average intensity and on $Q$ ). This Gaussian central-limit theorem argument breaks down when the mean or variance of time between consecutive photon emissions diverges. Such a case corresponds to a Lévy type of behavior, which is relevant for the single quantum-dot experiments discussed in Section 8.

For the quantum-jump model under investigation and for zero detuning,

$$
\langle\tau\rangle \Gamma=\frac{\Gamma^{2}+2 \Omega^{2}}{4 \Omega^{2}}
$$

and

$$
Q=-\frac{6 \Omega^{2} \Gamma^{2}}{\left(\Gamma^{2}+2 \Omega^{2}\right)^{2}}
$$

based on Equation 48. These results were obtained by Mandel (113) using Equation 39. Thus $Q<0$. Owing to the Rabi oscillations, the emission process is not Poissonian, instead the photons are correlated. If photons are strongly correlated, $f(t)=\delta\left(t-\tau_{0}\right)$ and $Q=-1$. However, physically this limit cannot be reached. $Q$ attains its minimum when $\Omega=\Gamma / \sqrt{2}$, and then $Q_{\min }=-3 / 4$.

The results obtained here, although written in a slightly different form, are identical to those obtained previously by Mandel (113), Cook (108), and Lenstra (109). The method presented here simplifies the derivations based on Equation 39. The other methods, however, are used to justify the physical interpretation of the dynamical evolution of $|\Psi\rangle$ based on the non-Hermitian Hamiltonian (Equation $42)$. Finally, $\hat{f}(u)$ is related to other physical quantities, for example, $g^{(2)}(\tau)(28)$.

\subsection{Single Photons on Demand}

The possibility of generating single photons at predetermined times has attracted recent attention as it may be an important element for quantum computing and secure data transmission via quantum cryptography (114). The quantum-jump 
approach is a valuable tool in this direction because the approach can be used to model single-photon control using SM sources. Single-photon control from an SM source might lead to new insights into the field of deliberate quantum control (115).

Recent experiments by Brunel et al. (37) use the method of adiabatic following to prepare an SM in its fluorescing exited state. "Spontaneous emission from excited state gives rise to single photon. With our current experimental conditions, up to $74 \%$ of the sweeps lead to emission of a single photon" (35, p. 2722). The experiment shows that the SM (DBATT in an n-hexadecane matrix at $1.8 \mathrm{~K}$ ) performs as a reliable source for triggered photons.

Similar experiments, using different approaches, were performed in room temperature SMS $(110,117)$ and for single quantum-dot spectroscopy (118-121). Using $\pi$ pulse excitations, one may obtain a single photon per sweep, with a probability of one (i.e., when the length of the pulse is much shorter than the lifetime of the molecule).

Brunel et al. (37) used the quantum-jump approach to simulate the experimental data they obtained. The nearly perfect agreement between experiment and simulation is an indication that the quantum-jump approach, based on the picture of a two-level molecule, is a reasonable approximation in this case. These experiments (37) make use of the linear Stark effect. Using a radio-frequency (rf) field, the molecule's absorption frequency is varied in time $\omega=\omega_{0}+v \cos \left(\omega_{r f} t\right)$. In this way, the molecule is coming in and out of resonance with the exciting laser (frequency $\omega_{L}=\omega_{0}$ ). Brunel et al. (37) used the quantum-jump approach to calculate numerically the probabilities of emitting 0,1 , and 2 photons per sweep (the probability of obtaining three photons was very small).

Makarov \& Metiu (36) have investigated the control with an rf field of single photon emission from an SM source. Using numerical simulations based on the quantum-jump approach, they showed an interesting and nontrivial dynamical localization behavior. In the context of SMS the dynamical localization manifests itself as a sharp increase of the average time between successive emission events for specific nontrivial values of the rf-driving field. Interestingly, the theoretical problem in Reference 36 is related to laser-induced localization of a quantum particle in a symmetric double-well potential $(122,123)$.

The problem of single photon control opens many interesting unsolved theoretical questions: (a) How does one choose the best control parameters that would yield optimal control of photons (e.g., what is the minimum of $Q$ )? (b) During each sweep, what is the probability of emitting $0,1,2$, or more photons? (c) How does one extend the models to investigate molecular systems beyond the two-level molecule approximation?

\section{GENERATING FUNCTION APPROACH}

We briefly review the Zheng-Brown approach $(31,32)$, to photon statistics in SMS. The approach, based on a generating function formalism, captures both subPoissonian behavior (e.g., antibunching) and super-Poissonian behavior caused by 
spectral diffusion. As mentioned sub-Poissonian statistics and antibunching are usually observed in the short-time behavior of the $Q$ parameter (typical timescale is nanoseconds).

The information on photon statistics is contained in the moment generating function (31)

$$
2 \mathcal{Y}(s) \equiv \sum_{N=0}^{\infty} s^{N} P_{N}(t)
$$

where $P_{N}(t)$ is the probability of $N$ emission events in the time interval $(0, t)$. Generalized optical Bloch equations are used to obtain $\mathcal{Y}(s)$, for a chromophore with single excited and ground state (31)

$$
\begin{aligned}
\dot{\mathcal{U}}(s) & =-\frac{\Gamma}{2} \mathcal{U}(s)+\delta_{L}(t) \mathcal{V}(s) \\
\dot{\mathcal{V}}(s) & =-\delta_{L}(t) \mathcal{U}(s)-\frac{\Gamma}{2} \mathcal{V}(s)-\Omega \mathcal{W}(s) \\
\dot{\mathcal{W}}(s) & =\Omega \mathcal{V}(s)-\frac{\Gamma}{2}(1+s) \mathcal{W}(s)-\frac{\Gamma}{2}(1+s) \mathcal{Y}(s) \\
\dot{\mathcal{Y}}(s) & =-\frac{\Gamma}{2}(1-s) \mathcal{W}(s)-\frac{\Gamma}{2}(1-s) \mathcal{Y}(s) .
\end{aligned}
$$

These equations are exact within the rotating wave approximation and optical Bloch equation formalism. Similar to the previous Section, $\Gamma$ is the spontaneous emission rate of the electronic transition and $\Omega$ is the Rabi frequency. The time evolving detuning is $\delta_{L}(t)=\omega_{L}-\omega_{0}-\omega(t)$, where $\omega_{L}$ is the laser frequency, $\omega_{0}$ is the molecule's bare frequency, and $\omega(t)$ is the stochastic spectral diffusion process.

Solution of Equation 60 yields, in principle, $\mathcal{Y}(s)$ from which the moments of $N$

$$
\langle N(t)\rangle_{\mathrm{QM}}=\left.2 \frac{\partial \mathcal{Y}(s)}{\partial s}\right|_{s=1}\left\langle N^{2}(t)\right\rangle_{\mathrm{QM}}=\left.2 \frac{\partial^{2} \mathcal{Y}(s)}{\partial s^{2}}\right|_{s=1}+\left.2 \frac{\partial \mathcal{Y}(s)}{\partial s}\right|_{s=1},
$$

are obtained. With Equation 61 the Mandel $Q$ parameter can be obtained. Two types of averaging are involved in this problem: averages with respect to the photon emission process (denoted with $\langle\cdots\rangle_{\mathrm{QM}}$ ), and also averages with respect to the spectral diffusion process.

The main questions concerning Equation 60 are as follows: (a) What is the physical meaning and foundations of the generalized optical Bloch equations? (b) What are the method of solutions of the equations (32)? (c) What is the meaning and procedure of averaging over the spectral diffusion process and the stochastic emission process in the context of SMS (this issue was not treated yet in depth)? (d) What are characteristic behaviors of the $Q$ parameter, for spectral diffusion processes, specifically (124)? (e) When does $Q$ yield sub-Poissonian behavior? $(f)$ How can we maximize the sub-Poissonian nature of the emission process (i.e., 
how to choose $\Omega$ ) (124)? The latter point $(f)$ is important for the efficient detection of quantum effects in SMS because choosing values of the Rabi frequency that are too small or too large results in very small, and hence, undetectable values of $Q$.

The physical meaning of $\mathcal{U}(s), \mathcal{V}(s)$, and $\mathcal{W}(s)$ and their relation to the standard Bloch equation was given in Reference 31 , and in a closely related work by Mukamel (105). The generating function formalism is mathematically equivalent to other approaches to photon statistics developed in quantum optics (108), for example, the quantum-jump formalism (28).

The behavior of the $Q$ parameter for a single molecule undergoing a spectral diffusion process, was investigated analytically only recently (124). Similar to Section 5, a telegraph type of spectral diffusion process where $\omega(t)=v h(t)$, and $h(t)=+1$ or $h(t)=-1$, with transition rate $R$ between state +1 and state -1 was choosen. An exact solution for the long-time behavior of the Mandel $Q$ parameter was obtained by He \& Barkai (124). The solution is valid for weak and strong laser fields, and gives the conditions on the spectral diffusion timescale and magnitude of spectral jumps for sub-Poissonian nonclassical behavior to be observed.

In the slow-modulation limit $R \ll v$ super-Poissonian behavior was obtained (124) $Q>0$, similar to the predictions of the semiclassical theory $(29,30)$ discussed in Section 5. Also Zheng \& Brown (31) used numerical simulation to show that the semiclassical approach works well in the slow-modulation limit. Most of the SM experiments are analyzed in a limit in which bath fluctuations are slow because these types of fluctuations are easier to detect experimentally.

In the fast-modulation limit $v \ll R$ a sub-Poissonian behavior is obtained (124), and a similar conclusion was obtained in Reference 32 on the basis of numerical simulations. The behavior of $Q$ is shown in Figure 8 . Strong sub-Poissonian behavior is observed for nonperturbative driving field $\Omega=\Gamma / \sqrt{2}$, implying that predictions of the linear response semiclassical theory are not valid in this case. The exact solution of Equation 60 yields in the limit $R \rightarrow \infty$ keeping $\Omega, \Gamma$, and $v$ fixed Mandel's result, Equation 58. As expected when the timescale for spectral jumps becomes very short, the molecule does not respond to the stochastic fluctuation. A more physically interesting case is to let $R \rightarrow \infty$ and $v \rightarrow \infty$ but keep the spectral diffusion contribution to the line width $\Gamma_{\text {eff }} \equiv v^{2} / R$ finite. In this limit the line shape is $(a)$ Lorentzian with a full width at half maximum equal to $\Gamma+\Gamma_{\text {eff }}$ (provided that the external fields are weak) and $(b)$ as shown in Figure 8 the line shape exhibits motional narrowing i.e., as $R$ is increased the line becomes narrower. In this limit (124)

$$
Q_{\text {fast }}=-\frac{2 \Gamma \Omega^{2}\left[3 \Gamma^{3}+5 \Gamma \Gamma_{\mathrm{eff}}^{2}+\Gamma_{\mathrm{eff}}^{3}-4 \Gamma \omega_{L}^{2}+\Gamma_{\mathrm{eff}}\left(7 \Gamma^{2}+4 \omega_{L}^{2}\right)\right]}{\left[\Gamma^{3}+\Gamma \Gamma_{\mathrm{eff}}^{2}+2 \Gamma \Omega^{2}+2 \Gamma_{\mathrm{eff}}\left(\Gamma^{2}+\Omega^{2}\right)+4 \Gamma \omega_{L}^{2}\right]^{2}},
$$

where the bare frequency of the molecule's electronic transition $\omega_{0}$ was set to zero. Note that photon statistics are sub-Poissonian provided that the detuning $\omega_{L}$ is not 
too large. The $Q$ parameter, for zero detuning, obtains its minimum when

$$
\frac{\Omega_{\min }}{\Gamma}=\sqrt{\frac{\left(\Gamma+\Gamma_{\text {eff }}\right)}{2 \Gamma}},
$$

and then $Q_{\min }=-\left(\Gamma_{\text {eff }}+3 \Gamma\right) / 4\left(\Gamma+\Gamma_{\text {eff }}\right)$. The simple Equation 63 shows how to choose the Rabi frequency in order to obtain strong sub-Poissonian behavior. Unlike $R$ and $v$, the parameter $\Gamma_{\text {eff }}$ is a physical observable, obtained in experiment from the width of the line shape, hence Equation 63 is of practical value. Finally we note that it would be interesting to obtain a quantum version of the generalized Wiener-Khintchine in Section 5, based on the linear response theory of Equation 60.

\section{FLUORESCENCE INTERMITTENCY AND STRANGE KINETICS}

Many SMS experiments exhibit fascinating fluorescent intermittency. The phenomenological and stochastic description of these fluctuations, for example, the fluorescence intermittency of single quantum dots $(125,126)$, is now considered. Quantum dots exhibit blinking behavior: At random times the quantum dot jumps between a bright state in which it emits many photons and a dark state in which it is turned off. The on (or off) state is believed to correspond to the neutral (or charged) state of the quantum dot (127). Thus statistics of on and off times teach us about the charging kinetics of the quantum dot.

Several examples of intensity fluctuations that are due to different physical mechanisms may be found in other SM systems. As mentioned in Section 4, intersystem crossing into a nonemissive triplet state causes triplet blinking (58, 134). Chemical dynamics can cause an SM to change its emissive state. For example, enzymatic turnovers of single cholesterol oxidase molecules have been observed in real time by monitoring the emission from the enzymes's active site (flavin adenine dinucleate) (63). Large and slow spectral shifts (i.e., shifts due to spectral diffusion) can cause the molecule to turn off and on when the exciting laser frequency is fixed. The latter behavior is also found in SMS in low-temperature glasses.

Unexpectedly, recent single quantum-dot experiments $(38,39,43)$ showed that distributions of on and off times follow a universal power-law behavior. The probability-density function of on and off times decay as $\psi\left(t_{o n}\right) \propto t_{o n}^{-\alpha_{o n}}$ and $\psi\left(t_{o f f}\right) \propto t_{o f f}^{-\alpha o f f}$. Brokmann et al. (43) measured 215 QDs, and the exponents $\alpha_{o n}$ and $\alpha_{\text {off }}$ were estimated to be $1.58 \pm 0.17$ and $1.48 \pm 0.15$, respectively. Using the Kolmogorov-Smirnov likelihood estimator, they concluded that the QDs are statistically identical.

The power-law behavior of the off time is temperature independent, found in different types of quantum dots (both CdSe and CdTe), and is independent of the radius of the quantum dot as well as the laser intensity. The on times exhibit similar 

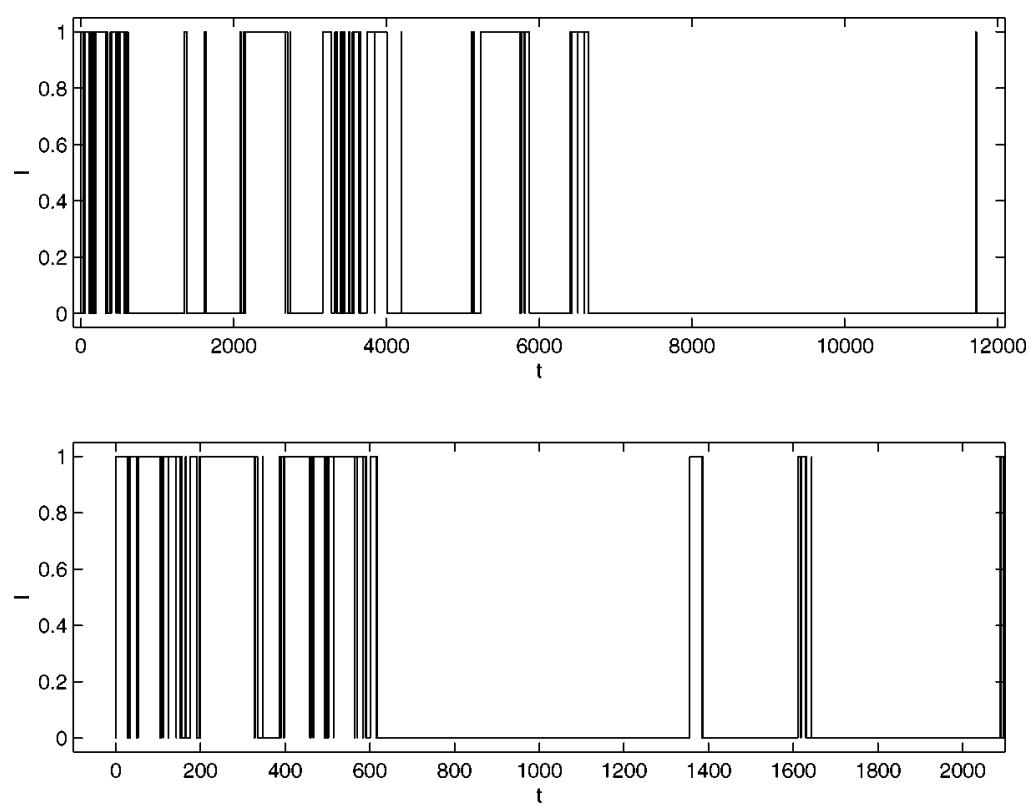

Figure 11 Blinking behavior of single quantum dots based on a two-state stochastic model. The intensity of the model quantum dot jumps between two states $I=1$ and $I=0$. The distribution of on times $(I=1)$ and off times $(I=0)$ is long tailed, in such a way that the averaged on and off times diverge. Such a behavior was observed in the experiments of Bawendi's (39) and Nesbitt's (38) groups. Notice the off-time interval $6600<t<11700$ (upper panel), which is of the order of the measurement time [time measured in arbitrary units]. The lower panel is a zoom of the upper panel for times $t<2100$. We used $\psi_{ \pm}(t) \propto t^{-3 / 2}$ and a simple computer program to generate this figure.

features; however, a secondary photoinduced mechanism introduces a cut-off in the probability-density function (PDF) of on times. The mentioned cut-off depends on the laser intensity and temperature; however, when these approach zero, the cut-off time seems to diverge. The power-law behavior indicates that these systems exhibit extremely large fluctuations (see below for more details). A single realization of intensity fluctuations in a two-state model quantum dot is shown in Figure 11.

The $t^{-3 / 2}$ behavior of the on and off times suggests that a simple one-dimensional random-walk mechanism may be responsible for the power-law behavior. For an ordinary one-dimensional random walk, the probability-density function of first passage times (from, say, $L$ to the origin) exhibits $P(t) \propto t^{-3 / 2}$ behavior. An electron or hole once ionized may perform a random walk, either in energy space or perhaps on the surface of the quantum dot. If such a random walk is one dimensional, the off times obey a $3 / 2$ power-law behavior. Similar mechanisms may also control the on times. 
Verberk et al. (129) suggested a random distribution of traps in the vicinity of the QD to explain the power-law behavior of off times. The model in Reference 129 predicts that $\alpha_{\text {off }}<1$; the exponent is then nonuniversal in the sense that it depends on the specific configuration of traps. Currently, further measurements are needed to clarify whether the exponents vary from one QD to the other (129) or are universal (43). The QD behavior is one example where experiments in SMS have yielded a surprising result that still requires detailed theoretical investigation.

Next, we analyze photon-counting statistics based on a two-state model. This approach is useful for describing both simple Poisson processes and power-law processes. The latter is related to Lévy statistics, whereas the former is described well by Gaussian statistics.

\subsection{Nonergodic, Nonstationary Dynamics: Lévy Walk Approach}

For a two-state intensity model $I(t)=I_{+}$and $I(t)=I_{-}$. In the quantum dot experiments, the positive state is on, whereas the negative state is off: $I_{-}=0$ (i.e., a neglecting background). For a more general case, corresponding to an SM jumping between two emission states, the positive and negative times are mutually independent; each is an identically distributed random variable. The PDF of the \pm times is $\psi\left(t_{ \pm}\right)$, and their Laplace transforms are

$$
\hat{\psi}_{ \pm}(s)=\int_{0}^{\infty} e^{-s t} \psi_{ \pm}(t) \mathrm{d} t
$$

For an experiment in which the number of photon counts is recorded in a given time interval $(0, T)$, what is the probability $P(n, T)$ of detecting $n$ photon counts? Mandel's counting formula (Equation 26) is rewritten as

$$
P(n, T)=\int_{0}^{\infty} P(W, T) \frac{W^{n}}{n !} \exp (-W) \mathrm{d} W,
$$

where

$$
W=\xi \int_{0}^{T} I(t) \mathrm{d} t
$$

Here $\xi$ is the detection efficiency, which, without loss of generality, may be set to 1 . $P(n, T)$ is the Poisson transform of $P(W, T)$, which, in principle, can be calculated from the statistical properties of the random process $I(t)$. If $I(t)$ is nonrandom and independent of time, the counting statistics are Poissonian. A general discussion of the relation between $P(W, T)$ and $P(n, T)$ is given elsewhere $[(87,128)$ and references therein]. In several $\mathrm{SM}$ experiments one may obtain direct information on $P(W, t)$ (when fluctuations are slow). However, when the experimental time resolution is not sufficient (e.g., fast blinking), analysis based on counting statistics might still be a useful tool. 
The two-state model under consideration can be mapped onto a simple randomwalk model. We imagine the intensity $I(t)$ is a one-dimensional Brownian-type of particle whose velocity is given by $I(t)$, which jumps between two-state values $I_{-}$and $I_{+}$. Then, according to Equation $66, W$ is the coordinate of the particle. Random-walk theory can be used to analyze this type of problem. Hence, in the long-time limit, we expect central-limit theorems (Gauss and Lévy) to play an important role.

Skinner \& Geva (19) considered Poisson jumps between these two states. Berezhkovskii et al. $(22,23)$ used a random-walk approach, developed by Weiss (130), and obtained a general expression for $P(W, T)$ Laplace-Fourier space and explicitly for a Poisson process. For such processes, the two-state model describes fluorescence intermittency of probes attached to single DNA $(131,132)$ and tRNA (133). Molski (134) investigated $P(n, T)$ for the Poisson process in the context of triplet blinking.

We emphasize that the power-law case considered in Reference 42 exhibits a statistical behavior different from that found in the Poissonian-jump models. The random on and off times from a single realization follow the sequence

$$
\left\{t_{o n}^{1}, t_{o f f}^{1}, t_{o n}^{2}, t_{o f f}^{2}, \cdots, t_{o n}^{n}, t_{o f f}^{n} \cdots\right\} .
$$

In Figures 12 and 13 we plot a "staircase" using $t_{\text {on }}$ for the length of each step and $t_{\text {off }}$ for the height of the step. For an identical number of steps the Poissonian and power-law processes exhibit different behaviors.

The characteristic function of the random variable $W$ is then written as a sum of four terms:

$$
P(k, T)=\sum_{m= \pm} \sum_{n= \pm} p_{m} P_{m n}(k, T)
$$

where $P(k, T)$ is the Fourier transform of $P(W, T), p_{m}$ is the probability that the process begins from state $m\left(p_{+}+p_{-}=1\right)$, and $P_{m n}(k, T)=\left\langle e^{i k W}\right\rangle_{m n}$ is the conditional characteristic function that is obtained by an average over the paths restricted to the state $m$ at the initial time $t=0$ and to the state $n$ at the final observation time $T$. This procedure yields (42)

$$
\begin{aligned}
P_{++}(k, T) & =\mathcal{L}^{-1}\left\{\frac{1-\hat{\psi}_{+}\left(s_{+}\right)}{s_{+}\left[1-\hat{\psi}_{+}\left(s_{+}\right) \hat{\psi}_{-}\left(s_{-}\right)\right]}\right\}, \\
P_{-}(k, T) & =\mathcal{L}^{-1}\left\{\frac{1-\hat{\psi}_{-}\left(s_{-}\right)}{s_{-}\left[1-\hat{\psi}_{+}\left(s_{+}\right) \hat{\psi}_{-}\left(s_{-}\right)\right]}\right\}, \\
P_{+-}(k, T) & =\mathcal{L}^{-1}\left\{\frac{\hat{\psi}_{+}\left(s_{+}\right)\left[1-\hat{\psi}_{-}\left(s_{-}\right)\right]}{s_{-}\left[1-\hat{\psi}_{+}\left(s_{+}\right) \hat{\psi}_{-}\left(s_{-}\right)\right]}\right\}, \\
P_{-+}(k, T) & =\mathcal{L}^{-1}\left\{\frac{\hat{\psi}_{-}\left(s_{-}\right)\left[1-\hat{\psi}_{+}\left(s_{+}\right)\right]}{s_{+}\left[1-\hat{\psi}_{+}\left(s_{+}\right) \hat{\psi}_{-}\left(s_{-}\right)\right]}\right\},
\end{aligned}
$$




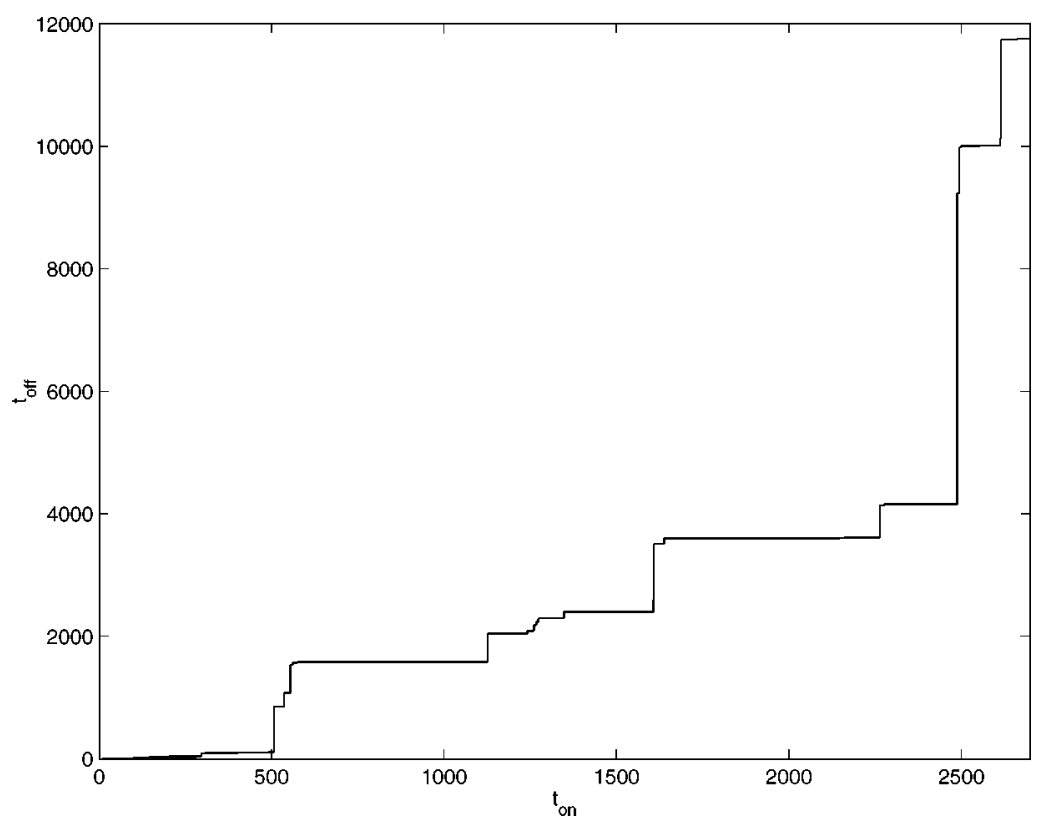

Figure 12 Power-law "staircase" for blinking quantum dots based on the two-state model. The height of each step is the off time, and the length of each step is the on time. There are 1000 steps in the figure. However, only a few large on and off times dominate the landscape of this staircase.

where $s_{ \pm}=s-i k I_{ \pm}$and $s$ is the Laplace variable.

We now consider the power-law case:

$$
\psi_{ \pm}(s)=1-A_{ \pm} s^{\alpha}+\cdots s \rightarrow 0,
$$

in which $\psi_{ \pm}(t) \propto t^{-(1+\alpha)}$ for $t \rightarrow \infty$ and $0<\alpha<1$. The quantum-dot case corresponds to $\alpha=1 / 2$. We assume the initial conditions $p_{ \pm}=A_{ \pm} /\left(A_{-}+A_{+}\right)$ and find in the long-time $T$ limit (42)

$$
\langle W\rangle \sim\left(p_{+} I_{+}+p_{-} I_{-}\right) T
$$

and

$$
\left\langle W^{2}\right\rangle-\langle W\rangle^{2} \sim(1-\alpha) p_{+} p_{-}\left(I_{+}-I_{-}\right)^{2} T^{2}
$$

Within the context of Lévy walks (146) the behavior in Equation 71 is called ballistic transport because the fluctuations exhibit a ballistic behavior $\left(\propto T^{2}\right)$ instead of the normal Gaussian behavior $(\propto T)$.

As a second example $\psi_{+}(t)=\psi_{-}(t)$. Here these sojourn-time probabilitydensity functions have a first finite moment, $\left\langle t_{+}\right\rangle=\left\langle t_{-}\right\rangle=\tau$, but a diverging 


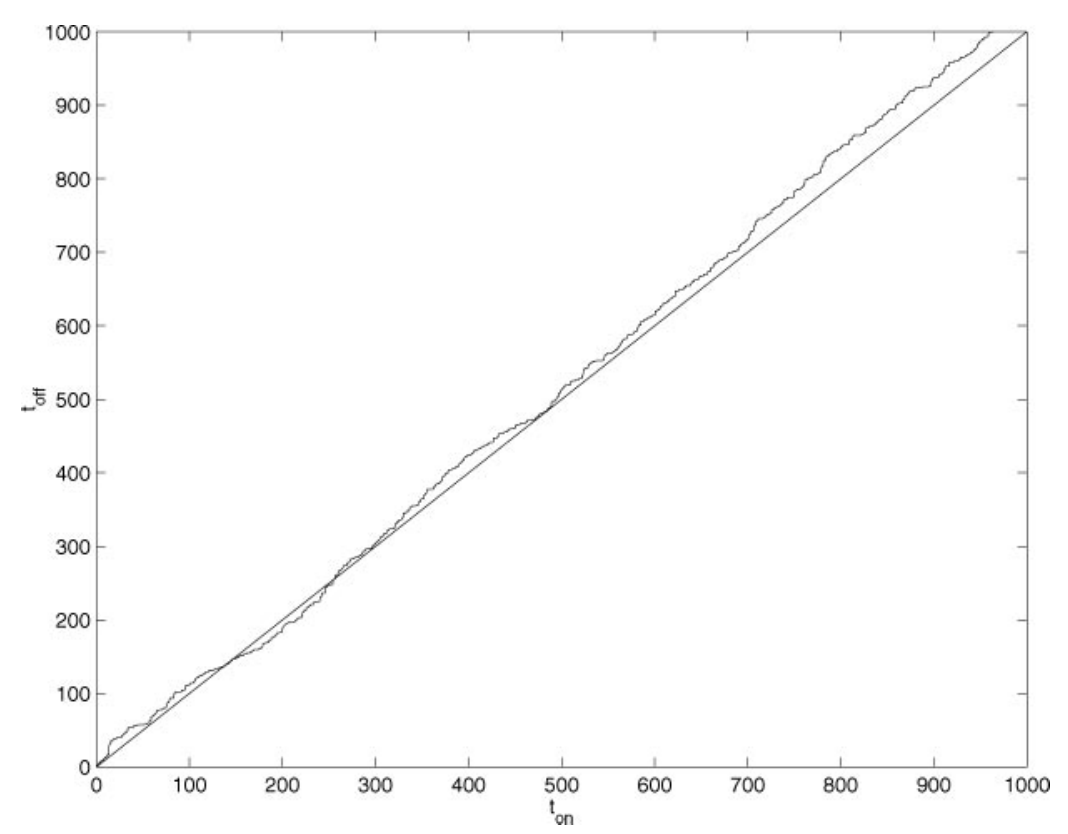

Figure 13 Normal "staircase" for exponential blinking. Compared with the corresponding blinking of the quantum dots, this staircase exhibits small fluctuations around the mean of many such staircases (i.e., the straight curve). There are 1000 steps in the figure.

second moment:

$$
\hat{\psi}_{ \pm}(s)=1-\tau s+A s^{\alpha}+\cdots, s \rightarrow 0,
$$

where $1<\alpha<2$. For simplicity $p_{+}=p_{-}=1 / 2$. In this case, we find in the long-time limit

$$
\langle W\rangle \simeq\left(\frac{I_{+}+I_{-}}{2}\right) T
$$

and

$$
\left\langle W^{2}\right\rangle-\langle W\rangle^{2} \sim \frac{A(\alpha-1)\left(I_{+}-I_{-}\right)^{2}}{2 \tau \Gamma(4-\alpha)} T^{3-\alpha},
$$

where $\Gamma(x)$ is the Gamma function. This type of behavior is called super-diffusion. When $\alpha \rightarrow 2$ the fluctuations tend to become linear in time (for $\alpha=2$ Lévy walks exhibit logarithmic corrections to the diffusive behavior). If $\alpha>2$, namely the case when the first two moments of the \pm times are finite, the fluctuations grow linearly with time. 
The photon statistics exhibit a behavior that is different from the standard photon-counting statistics, and $Q$ may increase with measurement time even for long times. Using Equation 29

$$
Q \propto \begin{cases}T & 0<\alpha<1 \\ T^{2-\alpha} & 1<\alpha<2 \\ T^{0} & 2<\alpha .\end{cases}
$$

This behavior is in contrast with traditional theories of photon-counting statistics that predict $Q \rightarrow T^{0}$ in the limit of long measurement time. The resulting fluctuations are extremely large if compared with the standard case corresponding to $\alpha>2$. One can show that the ballistic behavior of $Q(0<\alpha<1)$ is controlled by long sojourn of on and off times that are on the order of the measurement time $T$. The mean photon count always increases as $\langle n\rangle \sim T$ (see Equations 28, 70, and 73). Thus, $Q$, not $\langle n\rangle$ yields information on these unusual kinetics.

In terms of the nonergodic property of quantum dots, long measurement of the spectra of a single quantum dot does not yield an average equal to that found over an ensemble of chemically identical quantum dots. A time average over a single realization of a stochastic trajectory according to

$$
\overline{W^{n}(T)}=\frac{\int_{0}^{T} W^{n}(t) d t}{T}
$$

with $n=1,2, \ldots$ is different from the averaging denoted by $\langle\ldots\rangle$. For a process with $\alpha<1$ one will typically find a long time interval in which the system is in either a positive or a negative state; this time is on the order of the measurement time $T$. Thus, for two chemically identical quantum dots obeying this type of power-law dynamics, long-time averages over the two quantum dots are not generally identical, even in the limit $T \rightarrow \infty$. This is expected because we have no microscopic timescale in this problem.

The meaning of our averaging $\langle\ldots\rangle$ within an SM context is based on the following assumptions: Stochastic trajectories of the intensity fluctuation are recorded many times in a time interval $(0, T)$. The processes begins anew at time $t=0$ (i.e., the underlying stochastic process begins when the laser is turned on at start of observation $t=0$ ). Then averages over many such trajectories are computed to yield the average $\langle\cdots\rangle$. Finally, Verberk \& Orrit (135) have calculated the intensityintensity correlation function of the on-off process. However, their results are valid only for stationary processes that have finite averaged on and off times.

\section{SINGLE MOLECULES IN LOW-TEMPERATURE GLASSES}

In this section, we consider SMS in a disordered medium with special emphasis on SMS in low-temperature glasses. We use the approach of Geva \& Skinner (44), who modeled the line shape of SM in low-temperature glasses on the basis of the Kubo-Anderson sudden-jump approach to line-shape theory. The glass is modeled 
on the basis of the so-called standard tunneling model developed by Anderson, Halperin \& Varma (137) and independently by Phillips (138) in the early 1970s.

The standard tunneling model is a phenomenological model for low-temperature glasses (say, $T<3 \mathrm{~K}$ ). At its center is the concept of a two-level system (TLS). It is assumed that low-temperature excitations of glassy materials are two-level tunneling systems coupled to phonons. The tunneling parameters are distributed according to laws discussed below so as to mimic the disordered and complicated energy landscape of glass. Tunneling systems are randomly distributed in space with a low density. To a first approximation it is assumed that these tunneling systems interact with phonons but not with each other. Thus, the complicated glass is replaced with a simple noninteracting model. Brown \& Silbey (139) have partially justified the assumption of the noninteracting TLS for SMS in low-temperature glasses.

The standard TLS model was used successfully to predict macroscopic behaviors of many types of low-temperature glasses. For example, experiments have shown that low-temperature glasses exhibit a nearly linear dependence of the heat capacity with temperature, consistent with the standard tunneling model. The TLS approach was used successfully to model ensemble-averaged linear and nonlinear spectroscopy experiments. A fundamental first-principle understanding of TLS is still missing, although numerical simulations by Heuer \& Silbey (140) give some evidence of the microscopic nature of a few of these entities, whereas Lubchenko $\&$ Wolynes relate the TLS to motions of domain walls in the glass (141).

SMS can be used to investigate the assumptions of the standard tunneling model. The main questions in this direction are as follows: (a) Is the standard model valid? (b) How does one analyze the complex line shape behavior of an SM in a glass? (c) What is the physical nature of the TLS [e.g., can we control an individual TLS using, for example, the Stark effect and thus learn something of its microscopic origin (142)]? (d) Are TLS randomly distributed in the glass or do they preferentially appear at boundaries of clusters of atoms or molecules? $(e)$ What is the nature of the interaction between TLS and the probe SM? Because the spectra of SM are sensitive to their local nanoenvironments dynamics, SM experiments are used to investigate the dynamics of the glass and to start answering the above questions.

Beautiful experiments by Boiron et al. (52) showed how SMS may be used to investigate the validity of the tunneling model. The spectral trails of $70 \mathrm{SM}$ were recorded, and the data were compared with predictions based on the TLS picture of glass. Thirty percent of the SM behavior is not compatible with the standard model. Even though the number of molecules investigated is not large (owing to experimental difficulties) this method is a clear step forward in the pursuit of a better understanding of the dynamics of glasses. Recently, an analysis based on a larger sample of SM concluded that in a statistical sense the standard model predictions work well, although a few SM exhibited behavior not compatible with the standard model (48).

Let us briefly explain the main theoretical ideas behind the behavior of SM in a low-temperature glass and then consider some aspects of the problem in more detail. TLS are coupled to phonons in such a way that the state of the TLS changes 
with time. The state of the $n$th TLS is described by an occupation parameter, $\xi_{n}(t)$, which is equal to 1 or 0 if the TLS is in the ground or excited state, respectively. The stochastic process $\xi_{n}(t)$ is assumed to be a telegraph process described by up and down transition rates $K_{u}$ and $K_{d}$, respectively, which are related to each other by a detailed balance condition. When a TLS is excited by phonons, it shifts the absorption frequency of the molecule by $v_{n}$ (see below for further details).

Figures 2 and 3 exhibit model spectral trails of an SM coupled to a single TLS. In the slow modulation limit, the spectral trail follows the absorption frequency of the molecule. When a TLS is excited to its upper level $\left(K_{u}\right)$, or when it relaxes to its ground state $\left(K_{d}\right)$, we observe a frequency jump in the spectral trail [a typical timescale for spectral jumps observed using spectral trails in glasses is measured in seconds, although faster dynamics can be detected using $\left.g^{(2)}(\tau)\right]$.

The Orrit group $(70,71)$ investigated SM coupled strongly to a single TLS. Such behavior can be found when a TLS in the glass is in the vicinity of an SM in such a way that its frequency shift $v$ is much larger than the frequency shifts caused by other TLS in the system that serve as a background. In fact, such an SM-single TLS is not uncommon in low-temperature glasses. To determine whether the dynamical behavior of the SM is compatible with an interaction with a tunneling system, one needs to vary the temperature of the system. The temperature dependence of $K=K_{u}+K_{d}$ can be used to determine if the underlying process is a thermal activation or a phonon-assisted tunneling. In the experiments to date one finds behaviors $K(T) \propto T$ (one-phonon process) and $K(T) \propto T^{3}$ (two-phonon process). The case in which $K(T) \propto \exp (-\Delta E / T)$ (classical activation over barrier) was also found but only for one SM $(70,71)$.

When a TLS is excited it shifts the SM absorption frequency by $v_{n}$ (spectral jump). Thus, according to Kubo-Anderson's sudden-jump approximation the absorption frequency of the SM is

$$
\omega(\tau)=\omega_{0}+\sum_{n=1}^{N_{a c t}} \xi_{n}(\tau) v_{n},
$$

where $N_{a c t}$ is the number of active TLS and $\omega_{0}$ is the bare transition frequency. The summation (index $n$ ) is over randomly distributed TLS (see below for further details). The frequency shift due to the $n$th TLS is

$$
v_{n}=2 \pi \alpha \frac{\psi(\Omega) \Delta_{n}}{r^{3}},
$$

where $\alpha$ is a coupling constant, $\Delta_{n}$ is a dimensionless function of the internal parameters of the TLS, and $\Psi(\Omega)$ is a dimensionless function of order unity defined by the orientation of the $n$th TLS and the SM. $\Delta$ is given by $\Delta=A / E$. An assumption made in Equation 78 is that the SM/TLS interaction is dipolar, hence, the $1 / r^{3}$ interaction results.

Following previous findings (44) we generate the random environments and parameters as follows: Each TLS is characterized by its symmetry variable $A$ and 
tunneling element $J$, and the energy splitting of the TLS is $E=\sqrt{A^{2}+J^{2}}$. The probability of finding a TLS in its upper state $(\xi=1), p$, is given by a standard Boltzmann form $p=1 /\left\{1+\exp \left[E /\left(k_{\mathrm{b}} T\right)\right]\right\}$. The distribution of the asymmetry and tunneling parameters is $P(A) P(J)=N^{-1} J^{-1}$ for $J_{\min }<$ $J<J_{\max }$ and $0<A<A_{\max }$, where $A_{\max }, J_{\min }, J_{\max }$ are phenomenological parameters (given in Table 2), and $N$ is a normalization constant. The rate of the flipping TLS is $K=c J^{2} E \operatorname{coth}(\beta E / 2)$, where $c$ is the TLS phonon-coupling constant.

On the basis of Equations 77 and 78 one may, in principle, simulate the line shape or spectral trails of SM in low-temperature glasses, using the Bloch equation and Mandel's counting formula or using the quantum-jump approach (to date, however, no extensive simulations of spectral trails of an SM in a glass have been made). Because each molecule is in a unique environment, the spectral trail and line shape of each SM are also unique.

Geva \& Skinner (44) used such an approach to simulate line shapes of SM in glasses. They used the Wiener-Khintchine theorem (Equations 31 and 32) and generated random line shapes of one molecule at a time. The line shape exhibits complex behavior owing to the random environments each individual SM is situated in. The predictions in Reference 44 are in agreement with recent experiments shown in Figure 14. In their approach (44), the cutoff approximation was used. Thus in the simulation only TLS with jumping rates satisfying $K T>1$ were included. The idea is that if the jumping times are longer than measurement time $T$, then the jumping event of the TLS will not be observed in the experiment.

Plakhotnik (81) simulated line shapes in a time-dependent environment. $\mathrm{He}$ emphasized that taking a spectra of an SM in a glass (for one second), yields a random number of counts. Hence, repeating the measurement on an SM several times, i.e., taking snapshots, yields different line shapes for each snapshot. Thus the

TABLE 2 Parameter set for terrylene in polystryrene. See Reference 143, and the work of Geva \& Skinner (44) for further details

\begin{tabular}{lll}
\hline Parameter & Description & Value \\
\hline$T$ & Temperature & $1.7 \mathrm{~K}$ \\
$\tau_{\exp }$ & Experiment & $120 \mathrm{~s}$ \\
$A_{\max }$ & Maximal asymmetry & $17 \mathrm{~K}$ \\
$J_{\min }$ & Minimal tunneling element & $2.8 \times 10^{-7} \mathrm{~K}$ \\
$J_{\max }$ & Maximal tunneling element & $17 \mathrm{~K}$ \\
$r_{\max }$ & Maximal radial distance & $27.48 \mathrm{~nm}$ \\
$\rho$ & TLS density & $1.15 \times 10^{-2} \mathrm{~nm}^{-3}$ \\
$\alpha$ & SM/TLS coupling & $3.75 \times 10^{11} \mathrm{~nm}^{3} \mathrm{~Hz}$ \\
$c$ & TLS/phonon coupling & $3.9 \times 10^{8} \mathrm{~K}^{-3} \mathrm{~Hz}$ \\
\hline
\end{tabular}



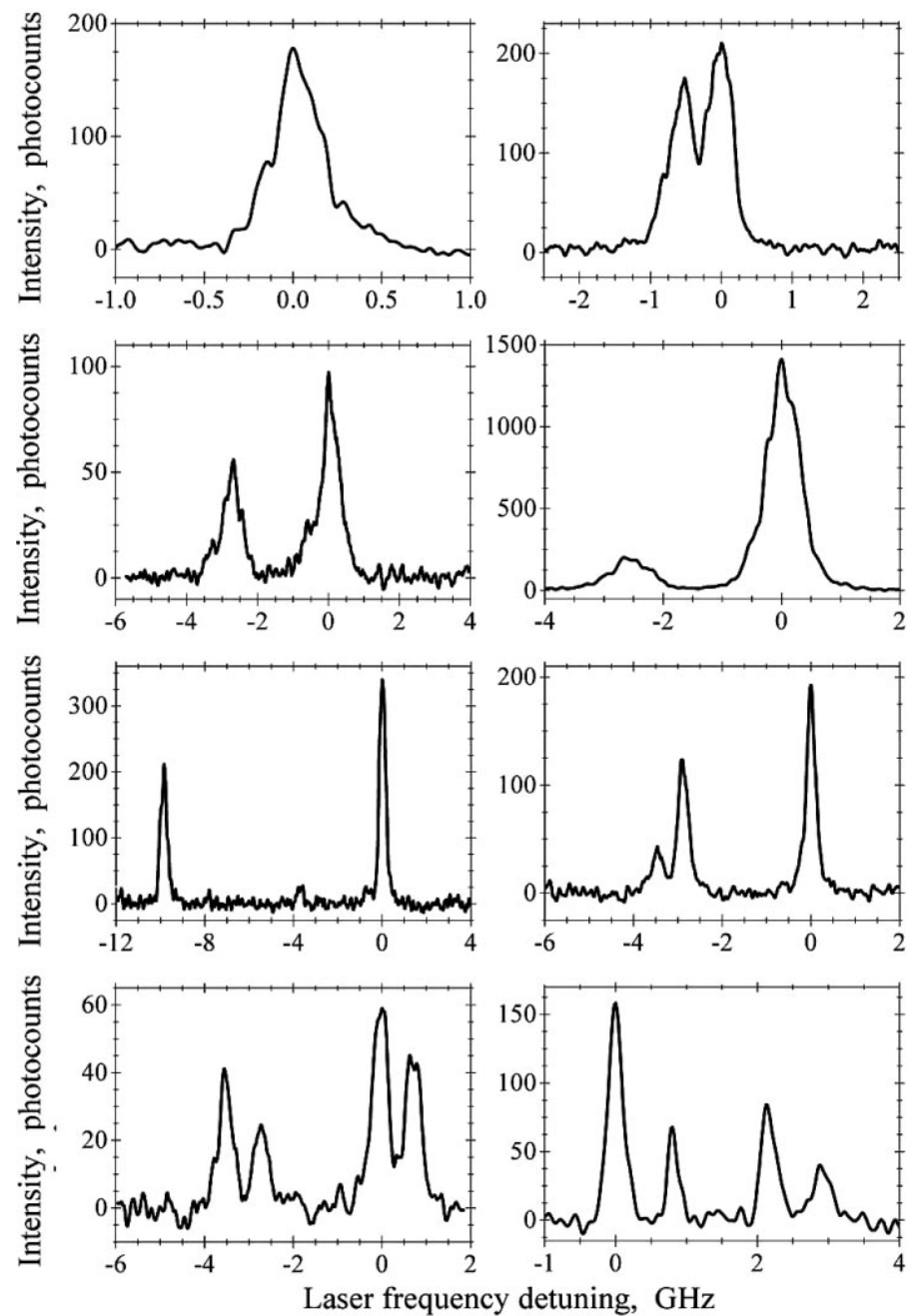

Figure 14 Line shapes of single tetra-tert-butylterrylene chromophores embedded in an amorphous polyisobutylene matrix, as measured by the Kador-Vainer group (48). Note the doublet and quartet features of some of the line shapes due to strong coupling to one or two TLS. One of the molecules has three peaks, indicating the possibility that this molecule is coupled to a three-level system. This rare type of behavior is not consistent with the standard tunneling model and the Geva-Skinner picture. Another possibility is that the measurement time was not long enough in this case and that in a longer measurement the molecule would be seen jumping between four states. Note that the analysis of the spectral trail histories allows us to distinguish between these possibilities. Following this line, we note that due to the nonergodicity of the glass increasing the measurement time might reveal different line shapes; thus the statistical properties of the line shapes in a glass generally depend on the timescale of the measurement. 
standard Wiener-Khintchine concept of averaged line shape breaks down. Plakhotnik objected to the approach by Geva and Skinner, claiming that the cutoff approximation is not justified. The cutoff approximation breaks down when $K T \simeq 1$, i.e., if the jumping time of a TLS $(1 / K)$ in the vicinity of the SM is of the order of measurement time $T$. The questions he raised (81) are not limited to the context of SMS in a glass. Because in SM measurement there is no averaging, the number of photon counts measured in any experiment is random. Thus the question is when one can use concepts like the Wiener-Khintchine theorem.

For glass we must distinguish between three kinds of perturbers (i.e., TLS). In fixing the measurement time $T$ we must demand that $K T \ll 1$ or $K T \gg 1$ for all the TLS in the vicinity of the SM for standard line-shape theory to hold. In effect, either the TLS jumps many times on the timescale of the measurement or the TLS is static (and then they do not contribute to the broadening of the line). If a TLS resides in the vicinity of the molecule with $K T \simeq 1$, and if the frequency shift due to this TLS is large, then standard line-shape theory is not valid. Using simulation one can estimate how many molecules in the glass belong to the category $K T \simeq 1$. However, this issue has not been investigated in detail. Thus it is still not clear how many SM will interact strongly with a TLS whose jumping timescale is of the order of a reasonable measurement time. Owing to a wide distribution of relaxation rates in glass, one cannot simply solve this problem by increasing the measurement time. We suspect that in a statistical analysis the number of such SM is not too large.

Finally, deviations between predictions based on simulations of the SM/glass model and measurement of SM line-width distribution have been recently reported (144). Good agreement between experiment and simulation was found by introducing an additional distribution of the coupling constant $\alpha$. The assumption of distribution of the coupling constant seems reasonable.

\subsection{Lévy Distribution of Line-Shape Cumulants}

The standard characterization of line shapes is based on their width and center location. This was used by Geva and Skinner to characterize line shapes in glasses. Such an approach is justified if the lines have simple shapes, i.e., all lines are Lorentzian or Gaussian with random widths. Hou et al. (145) used such an approach in their characterization of lines of SM in thin polymer films. However, as shown in Figure 14, the line shape of SM in low-temperature glasses is not a simple single-peaked function like a Lorentzian or a Gaussian. Its width at half maximum cannot be defined in many cases. Furthermore, the distribution of line widths gives only partial information on the complicated behavior of the lines. Instead of handling the problem of the distribution of a single parameter (e.g., the width), we are confronted with the problem of the distribution of the line shape (i.e., the distribution of a random function).

Barkai et al. (50) considered an infinite hierarchy of distribution functions to describe the behavior of the line shapes of SM in glasses. They suggested that each 
line be characterized by an infinite set of cumulants $\kappa_{1}, \kappa_{2}, \kappa_{3} \ldots$. Because each molecule is in a random environment, these cumulants are random variables. The PDFs $P_{1}\left(\kappa_{1}\right), P_{2}\left(\kappa_{2}\right) \cdots, P_{j}\left(\kappa_{j}\right), \cdots$ describe the statistical behavior of the lines. These distribution functions yield important information about the glass. They are also used to answer various questions. For example, are the TLS randomly distributed in space? Is the TLS/SM interaction dipolar (as suggested in Reference 44)? Is the standard model valid? Recently, data analysis of experiments based on this approach was made (48) (see details below).

Barkai et al. (50) showed that $P\left(\kappa_{j}\right)$ are stable Lévy PDFs, in the limit of slow modulation as defined by $K_{n} \ll v_{n}$. Thus in the slow-modulation limit Lévy's generalized central-limit theorem is valid. Using numerical simulations they (50) showed that Lévy statistics yields a good approximation to the distribution of line-shape cumulants, thus justifying the assumption of slow modulation (150).

Also, when all TLS are in the fast-modulation limit, a case that seems irrelevant for glasses, all lines exhibit simple behavior. Such lines are Lorentzian with a random center and a random width at a half maximum (50). The distribution of the center location and of the line widths also obeys Lévy statistics. A transition from simple line-shape behavior (i.e., Lorentzian line shapes, fast modulation) to complex line-shape behavior (i.e., multipeaked line shapes, splitting, slow modulation) of SM in disordered media has been investigated (50).

We now consider the theory of distribution of line-shape cumulants in some detail and discuss its relation to Lévy statistics. In terms of the sum of $n$ independent identically distributed random variables $\sum_{i=1}^{n} x_{i}$, if the variance of $x_{i}$ is finite, then the scaled sum converges in the limit of large $n$ to a Gaussian distribution. When the variance of $x_{i}$ diverges, the scaled sum converges in a limit to a Lévy-stable law, which is a natural generalization of the Gaussian (102). There are many physical applications of Lévy statistics in different fields of research (146-149).

The Fourier $x \rightarrow k$ transform of a Lévy-stable probability density $L_{\gamma, \eta}(x)$ is of the form

$$
\ln \left[\hat{L}_{\gamma, \eta}(k)\right]=-z_{\gamma}|k|^{\gamma}\left[1-i \eta \frac{k}{|k|} \tan \left(\frac{\pi \gamma}{2}\right)\right],
$$

where $0<\gamma \leq 2$ is called the characteristic exponent, $-1 \leq \eta \leq 1$ is the index of symmetry, and $z_{\gamma}>0$ is a scale parameter.

The cumulants of the random line shapes of SM have been derived (46), and they are given here in Table 3. To derive the cumulants, one uses the Wiener-Khintchine theorem (Equation 32) for the line where correlation function is calculated using Equations 31,77 , and 78 . No averaging over disorder is made to derive the cumulants in Table 3.

As previously mentioned, the cumulants are random numbers because they depend on the local nanoenvironment of the SM.

$\langle\cdot\rangle_{r \Omega A J}$ denotes an averaging over the random TLS parameters. The characteristic function of the odd $j$ cumulant, in the slow-modulation limit is given by (50), 
TABLE 3 Cumulants $\kappa_{j}$ of the SM line shape*

\begin{tabular}{ll}
\hline $\mathbf{j}$ & $\kappa_{j}$ \\
\hline 1 & $\sum_{n} p_{n} v_{n}$ \\
2 & $\sum_{n} p_{n}\left(1-p_{n}\right) v_{n}^{2}$ \\
3 & $\sum_{n} p_{n}\left(1-p_{n}\right)\left(2 p_{n}-1\right) v_{n}^{3}+i \sum_{n} p_{n}\left(1-p_{n}\right) K_{n} v_{n}^{2}$ \\
4 & $\sum_{n} p_{n}\left(-1+p_{n}\right)\left[K_{n}^{2}+v_{n}^{2}\left(-1+6 p_{n}-6 p_{n}^{2}\right)\right] v_{n}^{2}-$ \\
& $2 i \sum_{n} K_{n}\left(-1+p_{n}\right) p_{n}\left(-1+2 p_{n}\right) v_{n}^{3}$ \\
\hline
\end{tabular}

${ }^{*} p_{n}$ is the occupation probability of the $n$th TLS, $v_{n}$ is the frequency shift Equation 78 and $K_{n}$ is the flipping rate of the TLS. See Reference 50 for further details.

$$
\left\langle\exp \left(i k \kappa_{j}\right)\right\rangle_{r \Omega A J}=\hat{L}_{\gamma, 0}(k),
$$

with characteristic exponent

$$
\gamma=1 / j
$$

and a scale parameter $z_{\gamma}$ (50). The probability-density function of the jth cumulant is the inverse Fourier transform of Equation 80; hence, it is a Lévy-stable probability-density function. This Lévy behavior is not sensitive to the details of the model such as the exact distribution of tunneling parameter. $\gamma$ depends only on the dimension $d$ of the problem ( $d=3$, in our case) and the interaction exponent $\delta$ in $v \sim r^{-\delta}$ (from Equation 78; $\delta=3$, in our case). To derive Equation 80 we have used the assumption of independent TLS uniformly distributed in the system. For even cumulants and $0<\gamma<1$ we find

$$
\left\langle\exp \left(i k \kappa_{j}\right)\right\rangle_{r \Omega A J}=\hat{L}_{\gamma, \eta}(k),
$$

with a Lévy index of symmetry $\eta$ (50). Unlike the exponent $\gamma$ (Equation 81), the exponent $\eta$ depends on details of the model and on many parameters such as temperature (see Reference 50).

A comparison between the analytical predictions and a numerical simulation has been performed (50). The main question is whether the assumption of slowmodulation limit used in the derivation of analytical formulas is reasonable. In Figures 15 and 16 we show the model predictions for the first three cumulants. The figures show that Lévy statistics yields a good approximation for the numerical results.

The appearance of Lévy statistics in this context is due to the long-range interaction between the SM and the TLS. Briefly, the frequency shift $v$ can become very large (TLS in the vicinity of the SM) or small (TLS far from the SM). For power-law interaction (dipole interaction is an example) this leads to a Lévy type of behavior. Had the TLS/SM interaction been short ranged, the distribution of 


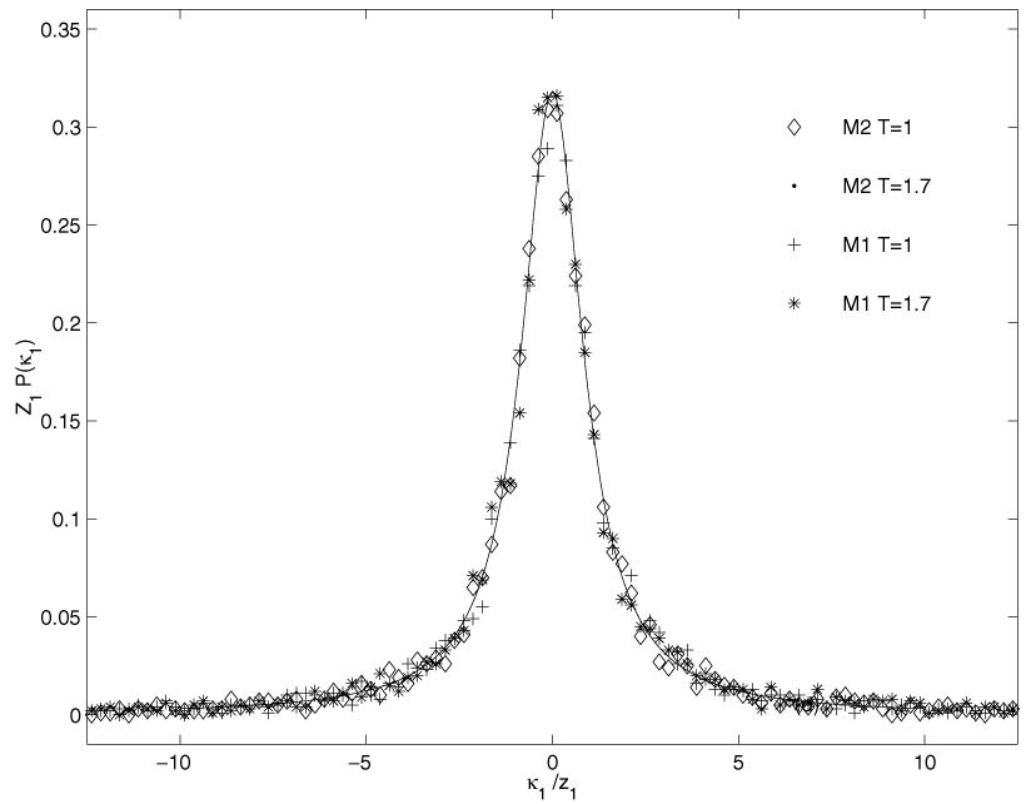

Figure 15 Scaled probability density of the first cumulant $P\left(\kappa_{1}\right) z_{1}$ versus $\kappa_{1} / z_{1}$. Symbols are the simulation results obtained from 4000 molecules for different cases indicated in the figure. The theory, plotted as a solid curve, predicts a Lorentzian density $P\left(\kappa_{1}\right)=L_{1,0}\left(\kappa_{1}\right)$ with a scaling parameter $z_{1}$, which varies from one set of data to the other. $M 1$ and $M 2$ are two slightly different models for the TLS/SM system (discussed in Reference 50).

the cumulants would be Gaussian-like. The importance of Lévy-stable laws in systems with long-range interactions, although usually overlooked, is not limited to the problem of SM in a glassy environment. Long-range interaction models of spectral diffusion $(151,152)$ and inhomogeneous line broadening (56) belong to the domain of attraction of Lévy statistics (see References 46 and 136 for further details).

Recent experiments analyzed by Barkai et al. (48) demonstrated that the statistical behavior of random line shapes of 244 single tetra-tert-butylterrylene chromophores embedded in an amorphous polyisobutylene matrix at $T=2 \mathrm{~K}$ is compatible with Lévy statistics and the theoretical predictions based on the standard tunneling model. The experimental results for the distribution of the first two cumulants are shown in Figures 17 and 18. In Reference 48 the ratio of the width of the Lévy distributions in Figures 17 and 18 is shown to be universal, in the sense that it does not depend on the coupling of the molecule to the TLS. This universal amplitude ratio is used to test the predictions of the standard model (see Reference 48 for details). 


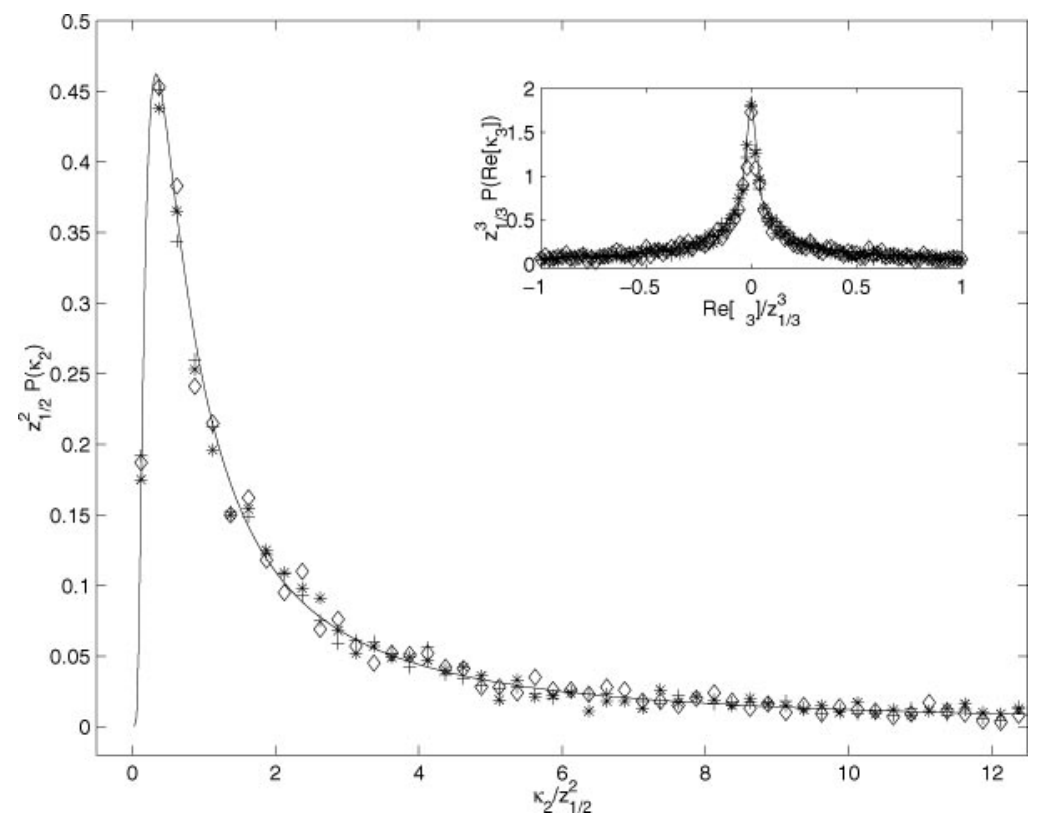

Figure 16 Scaled probability density of the second cumulant. $P\left(\kappa_{2}\right) z_{1 / 2}^{2}$ versus $\kappa_{2} / z_{1 / 2}^{2}$. The solid curve is Smirnov's density $L_{1 / 2,1}\left(\kappa_{2}\right)$. The inset shows the same theory plotted in Figure 15 but for $\operatorname{Re}\left[\kappa_{3}\right]$. The solid curve is a Lévy density $L_{1 / 3,0}\left(\operatorname{Re}\left[\kappa_{1 / 3}\right]\right)$.

\section{SUMMARY AND CONCLUSIONS}

The merit of SMS is that it removes the need for ensemble averaging. Hence, one has direct access to temporal fluctuations and fluctuations due to distribution of local nanoenvironments. The relation between photon-counting statistics and underlying microscopic events occurring in the molecules embedded in their condensed phase environment is a fascinating theoretical subject. Here we have discussed five approaches to time-dependent fluctuations in SMS and calculated for each case the corresponding $Q$ parameter:

1. The most straightforward approach is the rate-equation approach. The analysis of photon statistics in triplet blinking yields information on the dynamics within the molecule, for example, the triplet lifetime.

2. A generalized Wiener-Khintchine approach has been used to investigate the relation between photon-counting statistics and spectral diffusion. This analysis is useful when dynamics of the environment is investigated. In such a case the SM is a probe for the nanoenvironment dynamics. SMS in lowtemperature glasses belongs to this class. Because the theory is based on semiclassical concepts, it yields super-Poissonian behavior $Q>0$. 


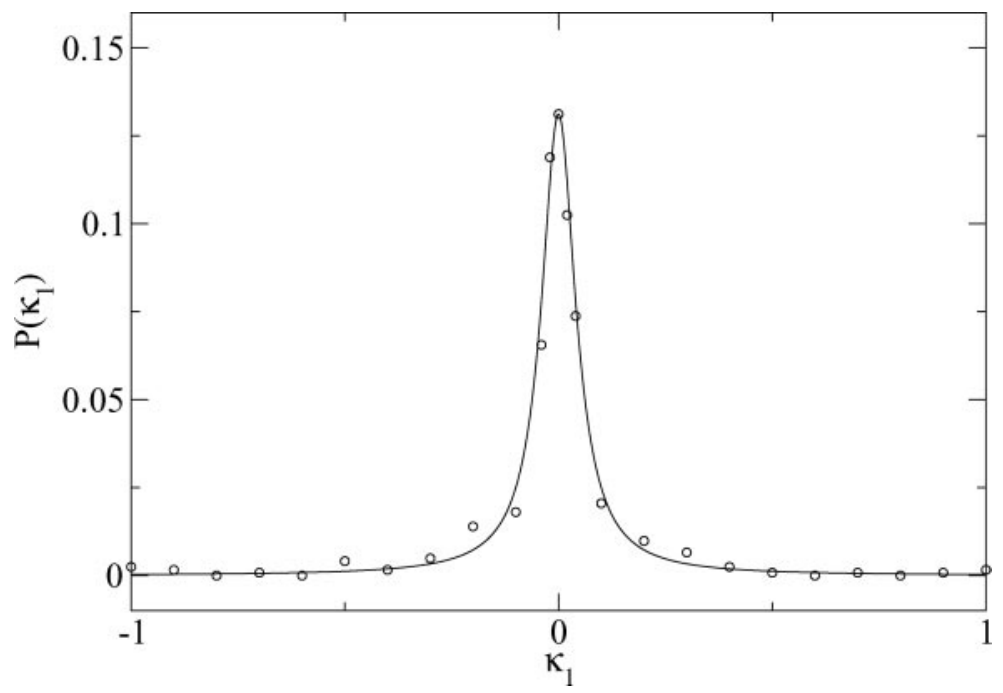

Figure 17 Experimental verification of theoretical predictions in Figure 15. Scaled probability density of the experimental results discussed in Reference 48 for the first cumulant $\kappa_{1}$ (units are $\mathrm{GHz}$ ). The dots represent experimental results of the VainerKador group, and the curve is a one-parameter fit to a symmetric Lévy probabilitydensity function with index 1, i.e., it is a Lorentzian.

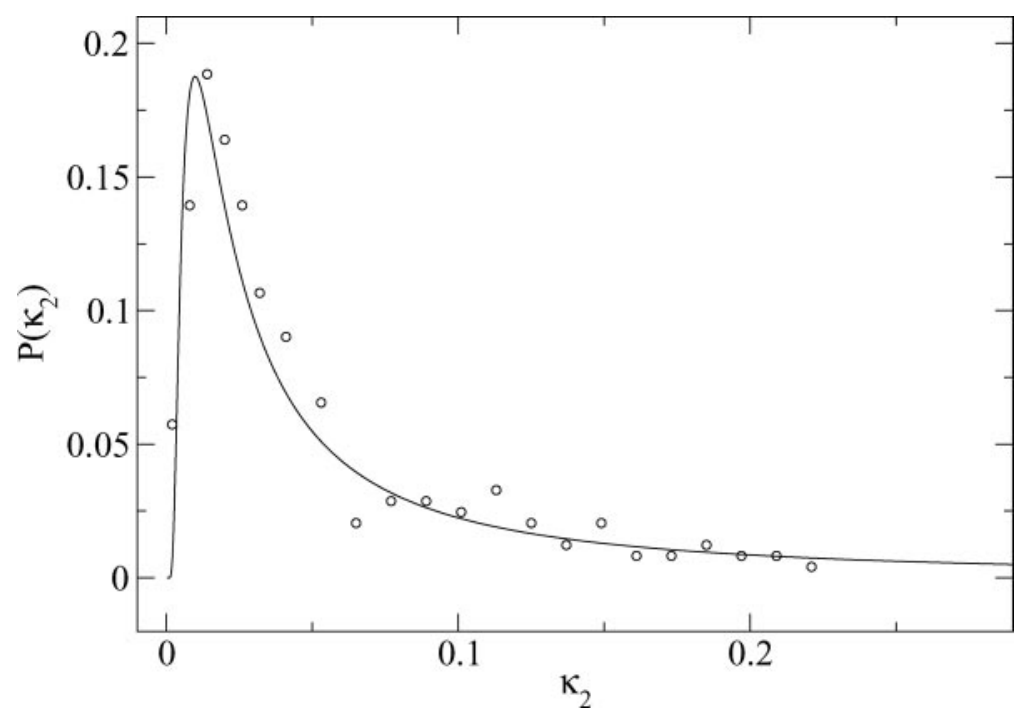

Figure 18 Experimental verification of theoretical predictions in Figure 16. Scaled probability density of the experimental results discussed in Reference 48 for the second cumulant $\kappa_{2}$ (units $\mathrm{GHz}^{2}$ ). The dots represent experimental results, and the curve is a one-parameter fit to a one-sided Lévy probability density with index $1 / 2$. 
3. The quantum-jump approach for SM sources yields the quantum mechanical description of a two-level SM interacting with a strong laser field. Unlike the semiclassical approach, this model yields negative values of $Q$ (87) (i.e., in the absence of spectral diffusion). Generalizations of this approach, beyond the two-level atomistic picture to different types of SM experiments seem important.

4. The generating function formalism captures both sub-Poissonian and superPoissonian behavior of SMS. For the problem of an SM undergoing a spectral diffusion process, the Mandel $Q$ parameter exhibits a transition between super- (slow process) to sub-Poissonian statistics (fast process). In most applications the solution to the generalized optical Bloch equations must be based on numerical methods (e.g., matrix inversion). These solutions yield probabilistic information (e.g., $P_{N}(t),\langle N(t)\rangle, Q$ etc). On the other hand the quantum-jump approach uses a numerical Monte Carlo strategy, in which photons are generated one at a time (28).

5. Finally, motivated by single quantum-dot experiments, we considered a Lévy-walk approach to SMS. This stochastic approach shows that $Q$ depends on the measurement time even in the long-time limit. This behavior is very different from existing theories for $Q$. These systems exhibit a nonergodic behavior and are fundamentally different from other SM systems.

The problem of line shapes in disordered materials (with an emphasis on line shapes in low-temperature glasses) has also been considered. Both theory and experiment have revealed the complexity of the line-shape structure of an SM in a low-temperature glass. The experiments in this field seem to confirm the validity of the phenomenological standard tunneling model. More generally, SMS in disordered materials leads to the problem of distribution of line shapes (or distribution of cumulants/moments of the line) as well as other parameters, for example, the distribution of lifetimes of SM. These distributions yield new insight into the disordered system (e.g., the power-law interaction between TLS and SM can be verified). They also lead to the need for new types of theories that go beyond the ensemble average.

In many cases, SMS experiments have revealed new types of dynamical behaviors that are still poorly understood theoretically: for example, $(a)$ the "cat's ears" resonances observed by Caruge \& Orrit (153) in an SM coupled to currents in semiconductors, $(b)$ the microscopic origin of the exponents $\alpha_{\text {on }}$ and $\alpha_{\text {off }}$ in quantum-dot experiments, $(c)$ the slow non-Markovian dynamics found in enzymatic turnovers of single cholesterol-oxidase molecules observed by Lu, Xun $\&$ Xie (63), and $(d)$ the distribution of relaxation times and sudden jumps of relaxation rates observed in SMS in a polymer system close to the glass transition (60). Although not discussed in this review, the theoretical understanding of SM experiments of macromolecules dynamics, using, for example, fluorescence 
resonance energy transfer, is an interesting topic of research (154-157). Because SMS unravels the ensemble average made in usual spectroscopic experiments, it is expected that this powerful technique will continue to reveal surprising results. We hope this review encourages theoretical studies that solve the existing enigmas of SMS.

\section{ACKNOWLEDGMENTS}

We thank M. Bawendi, Y. He, L. Kador, K. Kuno, A. Naumov, M. Orrit, K. Shimizu, Y. Vainer, and G. Zumofen for many helpful discussions on SMS. This work was supported by the NSF.

\section{The Annual Review of Physical Chemistry is online at http://physchem.annualreviews.org}

\section{LITERATURE CITED}

1. Moerner WE, Kador L. 1989. Phys. Rev. Lett. 62:2535-38

2. Orrit M, Bernard J. 1990. Phys. Rev. Lett. 65:2716-19

3. Moerner WE, Orrit MR. 1999. Science 283:1670-76

4. Rodrigues-Herzog R, Trotta F, Bill H. 2000. Phys. Rev. B 62:11163-69

5. Kurtsiefer C, Mayer S, Zarda P, Weinfurther H. 2000. Phys. Rev. Lett. 85:29093

6. Empedocles SA, Norris DJ, Bawendi MG. 1996. Phys. Rev. Lett. 77:3873-76

7. Xie XS, Trautman JK. 1998. Annu. Rev. Phys. Chem. 49:441-80

8. Weiss S. 1999. Science 283:167683

9. Moerner WE. 2002. J. Phys. Chem. B 106:910-27

10. Rhoades E, Gussakovsky E, Haran G. 2003. Proc. Natl. Acad. Sci. USA 100: 3197-202

11. Zhuang XW, Rief M. 2003. Curr. Opin. Struct. Biol. 13:88-97

12. Jia YW, Talaga DS, Lau WL, Lu HSM, DeGrado WF, Hochstrasser RM. 1999. Chem. Phys. 247:69-83

13. Deniz AA, Laurence TA, Beligere GS, Dahan M, Martin AB, et al. 2000.
Proc. Natl. Acad. Sci. USA 97:517984

14. Tamarat P, Maali A, Lounis B, Orrit M. 2000. J. Phys. Chem. A 104:116

15. Plakhotnik T, Donley E, Wild UP. 1997. Annu. Rev. Phys. Chem. 48:181-212

16. Xie XS. 2002. J. Chem. Phys. 117:1102432

17. Jung Y, Barkai E, Silbey RJ. 2002. J. Chem. Phys. 117:10980-95

18. Wang J, Wolynes P. 1995. Phys. Rev. Lett. 74:4317-20

19. Geva E, Skinner JL. 1998. Chem. Phys. Lett. 288:225-29

20. Schenter GK, Lu HP, Xie XS. 1999. J. Phys. Chem. A 103:10477-88

21. Chernyak V, Schulz M, Mukamel S. 1999. J. Chem. Phys. 111:7416-25

22. Berezhkovskii AM, Szabo A, Weiss GH. 1999. J. Chem. Phys. 110:914550

23. Berezhkovskii AM, Szabo A, Weiss GH. 2000. J. Phys. Chem. B 104:377680

24. Yang H, Xie XS. 2002. J. Chem. Phys. 117:10965-79

25. Barsegov V, Mukamel S. 2002. J. Chem. Phys. 117:9465-77 
26. Barsegov V, Mukamel S. 2002. J. Chem. Phys. 116:9802-10

27. Yang SL, Cao JS. 2002. J. Chem. Phys. 117:10996-1009

28. Plenio MB, Knight PL. 1998. Rev. Mod. Phys. 70:101-44

29. Barkai E, Jung Y, Silbey R. 2001. Phys. Rev. Lett. 87:207403

30. Jung Y, Barkai E, Silbey R. 2002. Adv. Chem. Phys. 123:199-266

31. Zheng YJ, Brown FLH. 2003. Phys. Rev. Lett. 90:238305

32. Zheng YJ, Brown FLH. 2003. J. Chem. Phys. 119:11814

33. Dalibard J, Castin Y, Molmer K. 1992. Phys. Rev. Lett. 68:580-83

34. Molmer K, Castin Y, Dalibard J. 1993. J. Opt. Soc. Am. 10:523-38

35. Makarov DE, Metiu H. 1999. J. Chem. Phys. 111:10126-36

36. Makarov DE, Metiu H. 2001. J. Chem. Phys. 115:5989-93

37. Brunel C, Lounis B, Tamarat P, Orrit M. 1999. Phys. Rev. Lett. 83:272225

38. Kuno M, Fromm DP, Hamann HF, Gallagher A, Nesbitt DJ. 2000. J. Chem. Phys. 112:3117-20

39. Shimizu KT, Neuhauser RG, Leatherdale CA, Empedocles SA, Woo WK, Bawendi MG. 2001. Phys. Rev. B 63:205316

40. Shimizu KT, Woo WK, Fisher BR, Eisler HJ, Bawendi MG. 2002. Phys. Rev. Lett. 89:117401

41. Messin G, Hermier JP, Giacobino E, Desbiolles P, Dahan M. 2001. Opt. Lett. 26: 1891-93

42. Jung Y, Barkai E, Silbey R. 2002. Chem. Phys. 284:181-94

43. Brokmann X, Hermier JP, Messin G, Desbiolles P, Bouchaud JP, Dahan M. 2003. Phys. Rev. Lett. 90:120601

44. Geva E, Skinner JL. 1997. J. Phys. Chem. B 101:8920-32

45. Mostovoy MV, Knoester J. 2000. J. Phys. Chem. B 104:12355-64

46. Jang SJ, Silbey RJ. 2003. J. Chem. Phys. 118:9324
47. Dempster SE, Jang SJ, Silbey RJ. 2001. J. Chem. Phys. 114:10015-23

48. Barkai E, Naumov AV, Vainer YG, Bauer M, Kador L. 2003. Phys. Rev. Lett. 91: 075502

49. Donley EA, Plakhotnik T. 2001. J. Chem. Phys. 114:9993-97

49a. Kubo R. 1954. J. Phys. Soc. Jpn. 6:935-44

50. Barkai E, Silbey R, Zumofen G. 2000. Phys. Rev. Lett. 84:5339-42

51. Naumov AV, Vainer YG, Bauer M, Zilker S, Kador L. 2001. Phys. Rev. B 63:212302$1-4$

52. Boiron AM, Tamarat $\mathrm{P}$, Lounis B, Brown R, Orrit M. 1999. Chem. Phys. 247:11932

53. Anderson PW. 1954. J. Phys. Soc. Jpn. 9:316-39

54. Kubo R. 1962. In Fluctuations Relaxation and Resonance in Magnetic Systems, ed. D ter Haar, pp. 23-68. New York: Plenum

55. Kubo R. 1969. Adv. Chem. Phys. 15:10127

56. Stoneham AM. 1969. Rev. Mod. Phys. 41:82-108

57. Bernard J, Fleury L, Talon H, Orrit M. 1993. J. Chem. Phys. 98:850-59

58. Yip WT, Hu D, Yu J, Vanden Bout DA, Barbara PF. 1998. J. Phys. Chem. A 102:7564-75

59. Veerman JA, Garcia-Parajo MF, Kuipers L, van Hulst NF. 1999. Phys. Rev. Lett. 83:2155-58

60. Deschenes LA, Vanden Bout DA. 2001. Science 292:255-58

61. Deschenes LA, Vanden Bout DA. 2001. J. Phys. Chem. B 105:11978-85

62. Lu HP, Xie XS. 1997. Nature 385:14346

63. Lu HP, Xun LY, Xie XS. 1998. Science 282:1877-82

64. Agmon N. 2000. J. Phys. Chem. B 104: 7830-34

65. Yang SL, Cao JS. 2001. J. Phys. Chem. B 105:6536-49

66. Schlegel G, Bohnenberger J, Potapova I, Mews A. 2002. Phys. Rev. Lett. 88: 137401 
67. Brown FLH. 2003. Phys. Rev. Lett. 90:028302

68. Ambrose WP, Moerner WE. 1991. Nature 349:225-27

69. Ambrose WP, Basche T, Moerner WE. 1991. J. Chem. Phys. 95:715063

70. Zumbusch A, Fleury L, Brown R, Bernard J, Orrit M. 1993. Phy. Rev. Lett. 70:358487

71. Fleury L, Zumbusch A, Orrit M, Brown R, Bernard J. 1993. J. Lumin. 56:1528

72. Bach H, Renn A, Zumofen G, Wild UP. 1999. Phys. Rev. Lett. 82:2195-98

73. Durand Y, Bloess A, Kohler J, Groenen EJJ, Schmidt J. 2001. J. Chem. Phys. 114:6843-50

74. Neuhauser RG, Shimizu KT, Woo WK, Empedocles SA, Bawendi MG. 2000. Phys. Rev. Lett. 85:3301-4

75. Mukamel S. 1995. Principles of Nonlinear Optical Spectroscopy. New York/ London: Oxford Univ. Press

76. Egorov SA, Stephens MD, Skinner JL. 1997. J. Chem. Phys. 107:1048591

77. Reilly PD, Skinner JL. 1993. Phys. Rev. Lett. 71:4257-60

78. Reilly PD, Skinner JL. 1995. J. Chem. Phys. 102:1540-52

79. Tanimura Y, Takano H, Klafter J. 1998. J. Chem. Phys. 108:1851-58

80. Geva E, Skinner JL. 1998. Chem. Phys. Lett. 287:125-30

81. Plakhotnik T. 1999. J. Lumin. 83:22128

82. Osad'ko IS, Yershova LB. 2000. J. Lumin. 86:211-17

83. Osad'ko IS, Khots EV. 2003. Opt. Spectrosc. 94:885-94

84. Bordat P, Brown R. 1998. Chem. Phys. Lett. 291:153-60

85. Osad'ko IS. 2001. J. Exp. Theor. Phys. 93:865-75

86. Bordat P, Brown R. 2002. J. Chem. Phys. 116:229-36

87. Mandel L, Wolf E. 1995. Optical Coher- ence and Quantum Optics. New York: Cambridge Univ. Press

88. Carmichael HJ, Singh S, Vyas R, Rice PR. 1989. Phys. Rev. A 39:1200-18

89. Molski A, Hofkens J, Gensch T, Boens N, De Schryver F. 2000. Chem. Phys. Lett. 318:325-32

90. Kimble HJ, Dagenais M, Mandel L. 1977. Phys. Rev. Lett. 39:691-95

91. Basche T, Moerner WE, Orrit M, Talon H. 1992. Phys. Rev. Lett. 69:1516-19

92. Fleury L, Segura JM, Zumofen G, Hecht B, Wild UP. 2000. Phys. Rev. Lett. 84: 1148-51

93. Hegerfeldt GC, Seidel D. 2003. J. Chem. Phys. 118:7741-46

94. Lounis B, Bechtel HA, Gerion D, Alivisatos P, Moerner WE. 2000. Chem. Phys. Lett. 329:399-404

95. Turro NJ. 1991. Modern Molecular Photochemistry. Mill Valley, CA: Univ. Sci. Books

96. Molski A. 2001. J. Chem. Phys. 114: 1142-47

97. Nonn T, Plakhotnik T. 2000. Phys. Rev. Lett. 85:1556-59

98. Lounis B, Moerner WE. 2000. Nature 407:491-93

99. Novikov E, Hofkens J, Cotlet M, Maus M, De Schryver FC, Boens N. 2001. Spectrochim. Acta Part A 57:2109-33

100. Cohen-Tannoudji C, Dalibard C. 1986. Europhys. Lett. 1:441-48

101. Cox DR. 1965. Renewal Theory. London: Methuen

102. Feller W. 1970. An Introduction to Probability Theory and Its Applications. New York: Wiley

103. Plakhotnik T. 1999. Phys. Rev. B 59:465860

104. Plakhotnik T, Wasler D. 1998. Phys. Rev. Lett. 80:4064-67

105. Mukamel S. 2003. Phys. Rev. A. Submitted. cond-mat/0307768 (http://arxiv.org/ list/cond-mat/0307768)

106. Wang ZS, Makarov DE. 2003. J. Phys. Chem. B 107:5617-22

107. Carmichael HJ. 1993. An Open System 
Approach to Quantum Optics. Berlin: Springer-Verlag

108. Cook RJ. 1981. Phys. Rev. A 23:124350

109. Lenstra D. 1982. Phys. Rev. A 26:336977

110. Lounis B, Jelezko F, Orrit M. 1997. Phys. Rev. Lett. 78:3673-76

111. Brunel C, Lounis B, Tamarat P, Orrit M. 1998. Phys. Rev. Lett. 81:267981

112. Hubner CG, Zumofen G, Renn A, Herrmann A, Mullen K, Basche T. 2003. Phys. Rev. Lett. 91:093903

113. Mandel L. 1979. Opt. Lett. 4:205-7

114. Turchete QA, Hood CJ, Lange W, Mabuchi H, Kimble HJ. 1995. Phys. Rev. Lett. 75:4710-13

115. Rice SA, Zhao M. 2000. Optical Control of Molecular Dynamics. New York: Wiley

116. Deleted in proof

117. Tinnefeld P, Mü C, Sauer M. 2001. Chem. Phys. Lett. 345:252-58

118. Petroff PM, Lorke A, Imamoglu A. 2001. Phys. Today 54:46-52

119. Michler P, Kiraz A, Becher C, Schoenfeld WV, Petroff PM, et al. 2000. Science 290:2282-85

120. Santori C, Pelton M, Solomon G, Dale Y, Yamamoto E. 2001. Phys. Rev. Lett. 86:1502-5

121. Zwiller V, Blom H, Jonsson P, Panev N, Jeppesen S, et al. 2001. Appl. Phys. Lett. 78:2476-78

122. Grossmann F, Ditrich T, Jung P, Hanggi P. 1991. Phys. Rev. Lett. 67:516-19

123. Bavli R, Metiu H. 1992. Phys. Rev. Lett. 69:1986-88

124. He Y, Barkai E. 2003. (http://arxiv.org/ abs/cond-mat/0312231)

125. Nirmal M, Dabbousi BO, Bawendi MG, Macklin JJ, Trautman JK, Harris TD. 1996. Nature 383:802-4

126. Banin U, Bruchez M, Alivisatos AP, Ha T, Weiss S, Chemla DS. 1999. J. of Chem. Phys. 110:1195-201

127. Efros EL, Rosen M. 1997. Phys. Rev. Lett. 78:1110-13
128. Mandel L, Wolf E. 1965. Rev. Mod. Phys. 37:231-87

129. Verberk R, van Oijen AM, Orrit M. 2002. Phys. Rev. B 66:233202

130. Weiss GH. 1994. Aspects and Applications of the Random Walk. Amsterdam: North Holland

131. Edman L, Mets U, Rigler R. 1996. Proc. Natl. Acad. Sci. USA 93:6710-15

132. Wennmalm S, Edman L, Rigler R. 1997. Proc. Natl. Acad. Sci. USA 94:7932-36

133. Jia Y, Sytnik A, Li L, Vladimirov S, Coopermann BS, Hochstrasser RM. 1997. Proc. Natl. Acad. Sci. USA 94:10641-46

134. Molski A. 2001. Chem. Phys. Lett. 324:301-6

135. Verberk R, Orrit M. 2003. J. Chem. Phys. 119:2214-22

136. Deleted in proof

137. Anderson PW, Halperin BI, Varma CM. 1972. Philos. Mag. 25:1-9

138. Phillips WA. 1972. J. Low. Temp. Phys. 7:351

139. Brown FLH, Silbey RJ. 1998. J. Chem. Phys. 108:7434-50

140. Heuer A, Silbey RJ. 1993. Phys. Rev. Lett. 70:3911-14

141. Lubchenko V, Wolynes PG. 2001. Phys. Rev. Lett. 87:195901

142. Bauer M, Kador L. 2003. J. Chem. Phys. 118:9069-72

143. Barkai E, Silbey R, Zumofen G. 2000. J. Chem. Phys. 113:5853-67

144. Donley EA, Bach H, Wild UP, Plakhotnik T. 1999. J. Phys. Chem. A 103:2282-89

145. Hou YW, Bardo AM, Martinez C, Higgins DA. 2000. J. Phys. Chem. B 104:212-19

146. Klafter J, Shlesinger MF, Zumofen G. 1996. Phys. Today 49:33-39

147. Bardou F, Bouchaud JP, Aspect A, CohenTannoudji C. 2002. Lévy Statistics and Laser Cooling. Cambridge, UK: Cambridge Univ. Press

148. Bouchaud JP, Georges A. 1990. Phys. Rep. 195:127-93

149. Metzler R, Klafter J. 2000. Phys. Rep. 339:1-77

150. Pfluegl W, Brown FLH, Silbey RJ. 
1998. J. of Chem. Phys. 108:6876- 154. Yang SL, Witkoskie JB, Cao JS. 2002. J. 83 Chem. Phys. 117:11010-23

151. Klauder JR, Anderson PW. 1962. Phys. Rev. 125:912-32

155. Chakrabarti D, Bagchi B. 2003. J. Chem. Phys. 118:7965-62

152. Zumofen G, Klafter J. 1994. Chem. Phys. Lett. 219:303-9

156. Lee CL, Lin CT, Stell G, Wang J. 2003. Phys. Rev. E 67:041905

153. Caruge JM, Orrit M. 2001. Phys. Rev. B 64:205202

157. Gopich IV, Szabo A. 2003. J. Phys. Chem. B 107:5058-63 

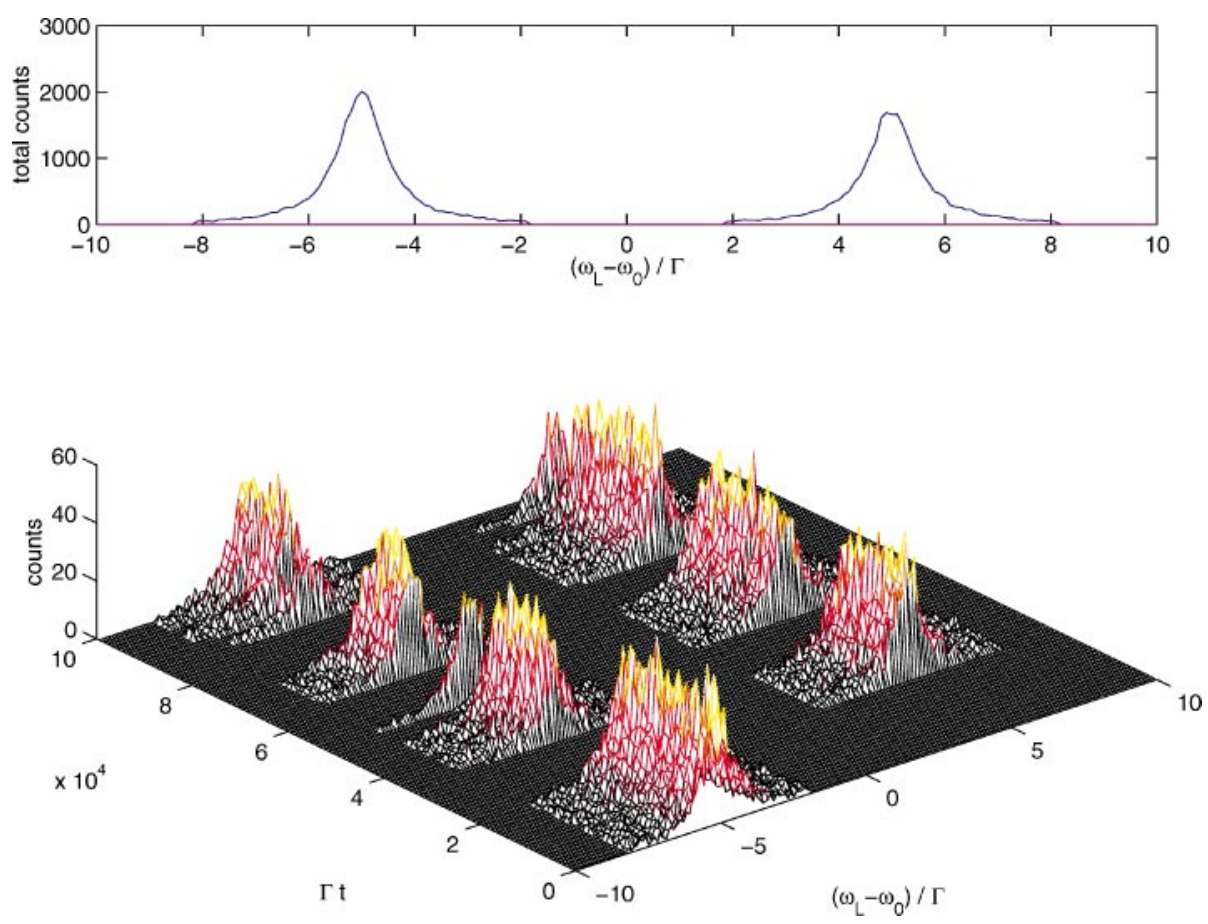

Figure 2 Spectral trail of an SM undergoing a slow spectral-diffusion process. The spectral trail mimics, for example, an SM coupled to a single TLS in a low-temperature glass (52). In the jump model, the absorption frequency jumps between two states, $\omega=\omega_{0}+v$ and $\omega=$ $\omega_{0}-v$, where $v=5 \Gamma$ and $\Gamma=1$. Here $R=10^{-4} \Gamma$; hence, the process is slow: $R \ll \Gamma \ll$ $v$. The spectral trail follows the absorption frequency of the molecule. The upper panel shows the total number of photon counts versus the laser frequency, i.e., the line shape of the molecule. 

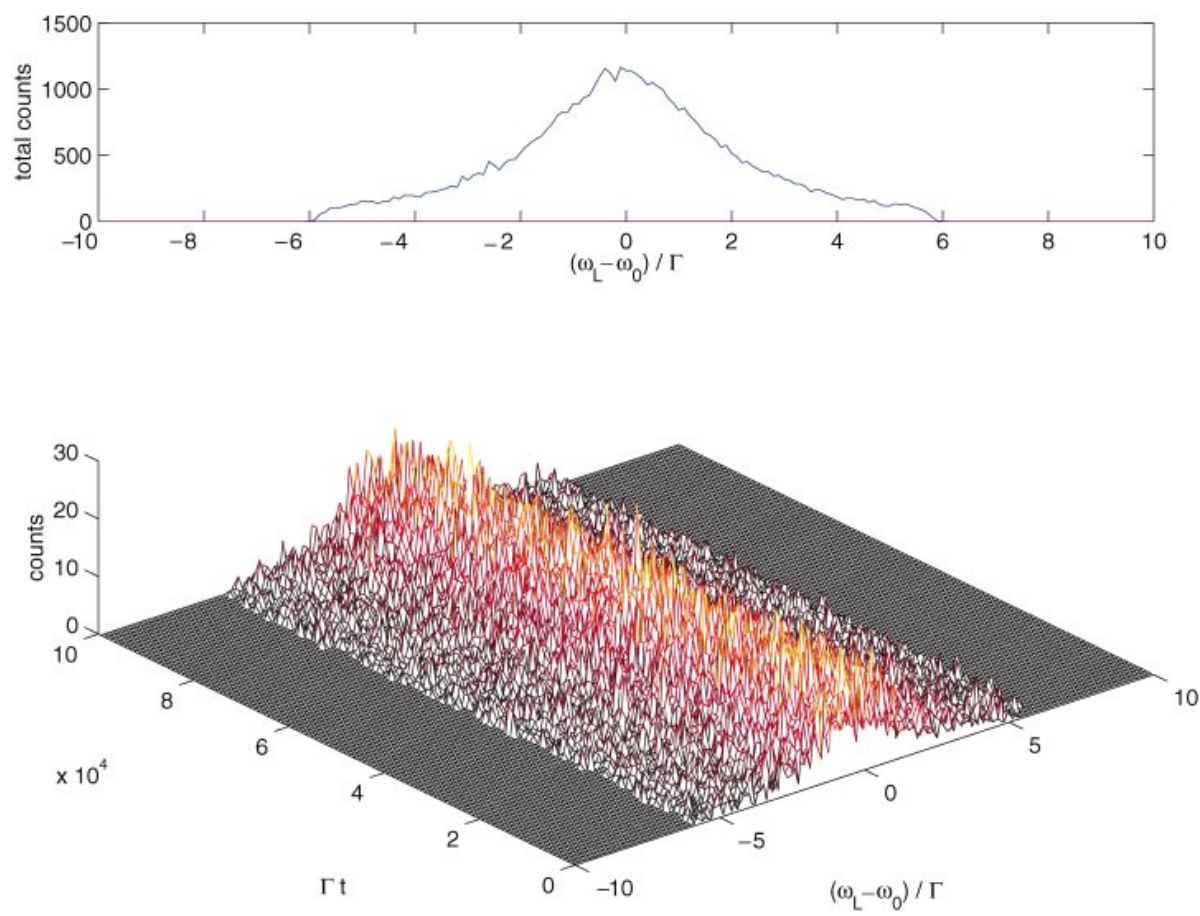

Figure 3 Spectral trail of an SM undergoing a fast spectral-diffusion process. $R=10 \Gamma$; while other parameters are the same as in Figure 2 hence, $\Gamma<v<R$. Owing to motional narrowing, the spectral trail does not follow the absorption frequency of the molecule i.e., $\omega=\omega_{0} \pm v$. Unlike the slow-modulation case, straightforward identification of the underlying rate and frequency jumps is impossible. However, careful analysis of the photon-counting statistics of such a process may lead to the determination of the underlying stochastic process and, hence, to the indirect detection of fast processes occurring in the condensed-phase SM system. 


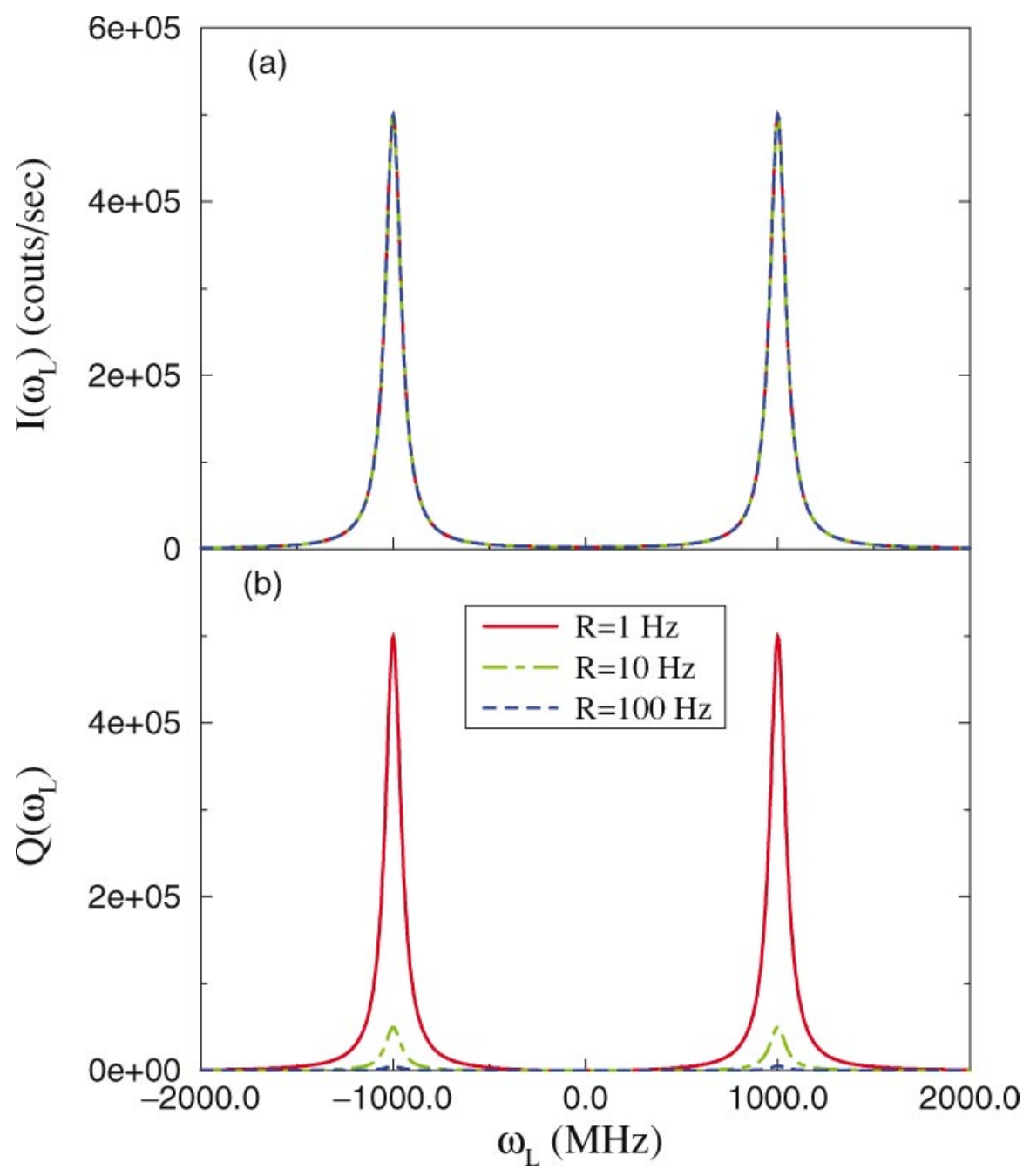

Figure 7 Mandel's parameter $Q$ and line shape $I\left(\omega_{\mathrm{L}}\right)$ in the slow-modulation limit, $R \ll \Gamma \ll$ $v$. The steady state, $T \rightarrow \propto$ limit is shown. In the slow-modulation limit, $Q$ is large compared with that in the fast-modulation limit. The figure mimics an SM coupled to a single TLS in a low-temperature glass. Parameters are $v=1 \mathrm{GHz}, \Gamma=100 \mathrm{MHz}, \Omega=\Gamma / 10$, and $R=1-$ $100 \mathrm{~Hz}$, for details see Reference 30 . In this limit $Q$ depends on the rate $R$, whereas $I\left(\omega_{L}\right)$ does not. Thus it is the fluctuations [i.e., $Q$ or $g^{(2)} \tau$ ] not the averaged line shape that yield insight into the underlying dynamics. $\omega_{L}$ is the detuning; $\omega_{0}=0$. 

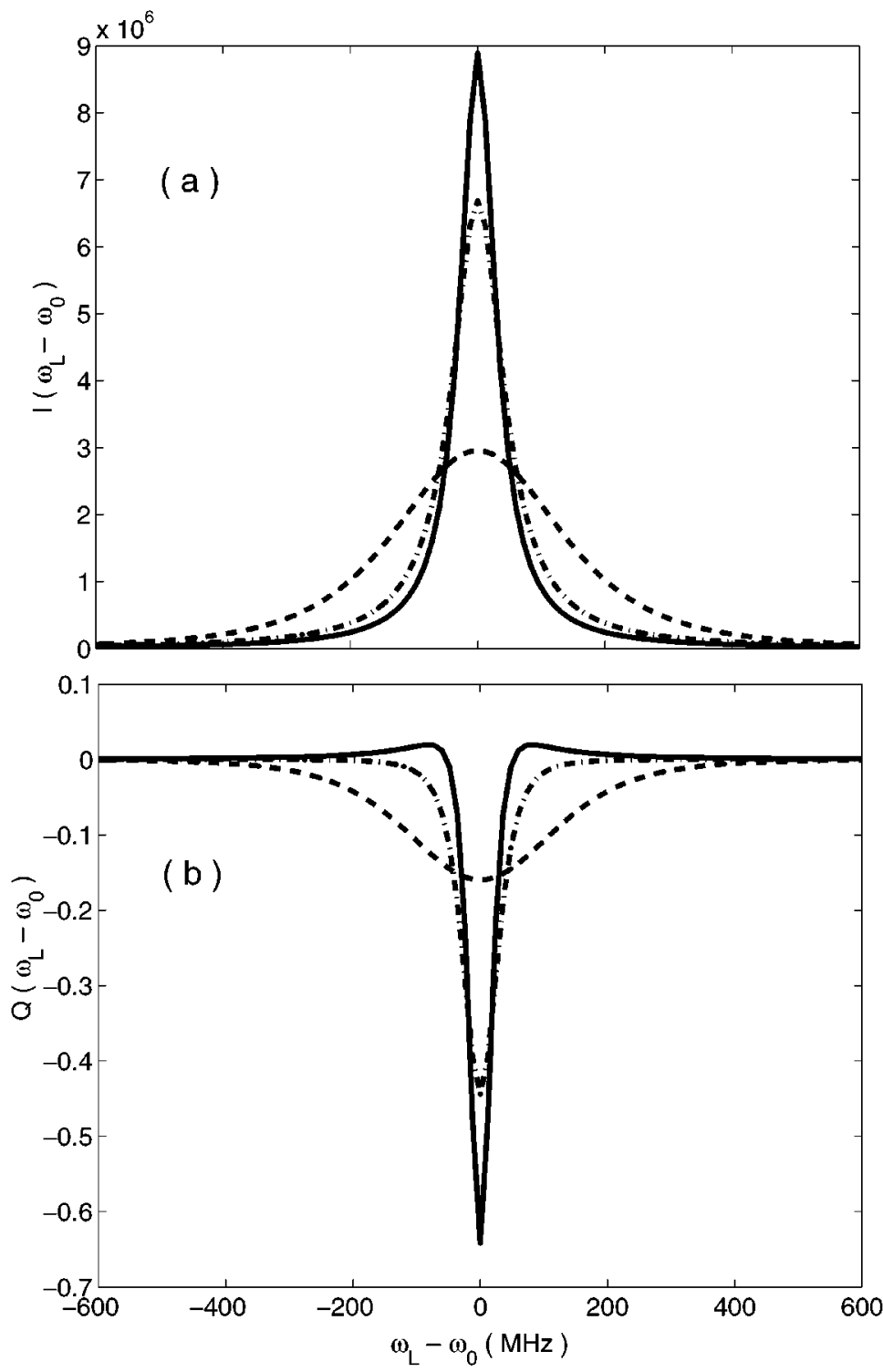

Figure $8 Q$ and $I\left(\omega_{L}\right)$ in the fast-modulation limit. The line exhibits motional narrowing: As the spectral diffusion process becomes faster, the line becomes narrower. The $Q$ parameter is calculated based on the generating function method Equation 62. $Q<0$ indicating sub-Poissonian behavior. As the processes become faster, the $Q$ parameter approaches Mandel's solution Equation 58. We used $\Gamma=40 \mathrm{MHz}, \Omega=\Gamma / \sqrt{2}, v=5 \Gamma$, and $R=5 \Gamma, 25 \Gamma, 100 \Gamma$ for dashed, dot-dashed, and solid lines respectively. 